\title{
ProCKSI: a decision support system for Protein (Structure) Comparison, Knowledge, Similarity and Information Daniel Barthel ${ }^{1}$, Jonathan D Hirst ${ }^{2}$, Jacek Błażewicz ${ }^{3,4}$, Edmund K Burke ${ }^{1}$ and Natalio Krasnogor*1
} \author{
Nottingham, Nottingham, NG7 2RD, UK, ${ }^{3}$ Institute of Bioorganic Chemistry, Polish Academy of Sciences, 61-704 Poznan, Poland and ${ }^{4}$ The \\ Institute of Computing Science, 60-965 Poznan, Poland \\ Email: Daniel Barthel - daniel.barthel@nottingham.ac.uk; Jonathan D Hirst - jonathan.hirst@nottingham.ac.uk; \\ Jacek Błażewicz - blazewic@put.poznan.pl; Edmund K Burke - edmund.burke@nottingham.ac.uk; \\ Natalio Krasnogor* - natalio.krasnogor@nottingham.ac.uk \\ * Corresponding author
}

Address: ${ }^{1}$ ASAP, School of Computer Science and IT, University of Nottingham, Nottingham, NG8 1BB, UK, ${ }^{2}$ School of Chemistry, University of

Published: 26 October 2007

BMC Bioinformatics 2007, 8:416 doi:10.1186/147|-2105-8-416
Received: 8 June 2007

Accepted: 26 October 2007

This article is available from: http://www.biomedcentral.com//47/-2/05/8/416

(c) 2007 Barthel et al; licensee BioMed Central Ltd.

This is an Open Access article distributed under the terms of the Creative Commons Attribution License (http://creativecommons.org/licenses/by/2.0), which permits unrestricted use, distribution, and reproduction in any medium, provided the original work is properly cited.

\begin{abstract}
Background: We introduce the decision support system for Protein (Structure) Comparison, Knowledge, Similarity and Information (ProCKSI). ProCKSI integrates various protein similarity measures through an easy to use interface that allows the comparison of multiple proteins simultaneously. It employs the Universal Similarity Metric (USM), the Maximum Contact Map Overlap (MaxCMO) of protein structures and other external methods such as the DaliLite and the TM-align methods, the Combinatorial Extension (CE) of the optimal path, and the FAST Align and Search Tool (FAST). Additionally, ProCKSI allows the user to upload a user-defined similarity matrix supplementing the methods mentioned, and computes a similarity consensus in order to provide a rich, integrated, multicriteria view of large datasets of protein structures.

Results: We present ProCKSI's architecture and workflow describing its intuitive user interface, and show its potential on three distinct test-cases. In the first case, ProCKSI is used to evaluate the results of a previous CASP competition, assessing the similarity of proposed models for given targets where the structures could have a large deviation from one another. To perform this type of comparison reliably, we introduce a new consensus method. The second study deals with the verification of a classification scheme for protein kinases, originally derived by sequence comparison by Hanks and Hunter, but here we use a consensus similarity measure based on structures. In the third experiment using the Rost and Sander dataset (RSI26), we investigate how a combination of different sets of similarity measures influences the quality and performance of ProCKSI's new consensus measure. ProCKSI performs well with all three datasets, showing its potential for complex, simultaneous multi-method assessment of structural similarity in large protein datasets. Furthermore, combining different similarity measures is usually more robust than relying on one single, unique measure.
\end{abstract}

Conclusion: Based on a diverse set of similarity measures, ProCKSI computes a consensus similarity profile for the entire protein set. All results can be clustered, visualised, analysed and easily compared with each other through a simple and intuitive interface.

ProCKSI is publicly available at http://www.procksi.net for academic and non-commercial use. 


\section{Background}

An important theme within structural bioinformatics is the analysis of protein sequences, the assessment of protein structural similarities and the inference of their biological functions. All of these play crucial roles in drug design and other structural inference activities [1] such as homology modeling and protein structure prediction. There, it is important to evaluate similarity among a large number of structures and to identify similar predictions or find the closest prediction to a given target [2].

Structural comparison and clustering is challenging, and effective algorithms continue to be introduced. The simplest global measure for protein structure comparison is the root mean square deviation (RMSD) [3,4]. More sophisticated methods are fragment matching $[5,6]$, geometric hashing [7], comparison of distance matrices [8], Monte Carlo (MC) algorithms or simulated annealing [8], maximum sub-graph detection [9], local geometry matching [10], incremental combinatorial extension (CE) of the optimal path [11], local global alignment (LGA) [12], dynamic programming [13-15], genetic algorithms (GA) [16], consensus shapes [17] or consensus structures [18], contact map overlaps (CMO) [19-25]), secondary structure matching (SSM) [26], memetic algorithms [27], or maximum clique detection [28,29]. In addition to these algorithms comparing two rigid protein structures, methods for flexible structure alignment have also been developed [30-32].

Many databases and web servers have been introduced implementing different concepts and aspects of the methodologies described above. An overview of recommended, well-tested resources, tools and databases for protein 3D structure and sequence comparison is given in the Bioinformatics Links Directory [33]. For more detailed information, the reader is referred to the overview articles of Galparin [34-36], and the webserver issues [37,38] and database issues [39-42] in Nucleic Acids Research.

\section{ProCKSI's Philosophy}

As it is evident from the list above, there are many biologically meaningful definitions of protein similarity. Several methods have been proposed and there is a variety of structure classification servers and databases available, each of them with its own interface, philosophy and, most importantly, biological conception of what "similarity" means. Paradoxically, the availability of all these methods with their unique interfaces and underlying biological hypotheses makes it more, and not less difficult for a structural biologist to decide which method to apply in which cases. Moreover, it is common to find papers related to protein structure comparison where the authors claim that their new method is better than another on a small set of test cases. These types of comparison can be mis- leading in at least two ways. First, changing the algorithm used to compare structures often inadvertently introduces a different comparison criterion, hence changing the problem itself. Secondly, the comparisons are usually done on a reduced number of data sets with characteristics that make them suitable to the new (implicit) criterion. In this paper we take the view that there is not one problem of protein structural comparison but rather many different, yet related, structural similarity problems where each of them might be best tackled with a different method. Hence, attempting to find the best method for protein structure comparison is a chimera. Instead, in line with other recent suggestions $[43,44]$ that the integration of a variety of feature detection techniques could enhance protein comparison, we advocate here an integrative approach that harnesses the best in each available method. This change in philosophy allows us to treat the assessment of protein structure comparisons as a decision support problem in which the task of the bioinformatician is to build up computer facilities that empower the user to make an informed decision with the minimum possible overhead. The advantage of this viewpoint is that it does not call for the abolition of one method in favour of another one but rather for the intelligent integration of every possible protein structure comparison method into one unified tool.

\section{ProCKSI's Core Protocol}

In this paper, we take the first steps towards the creation of an intelligent decision support system for protein structure comparison. We introduce a new meta-server for Protein (Structure) Comparison, Knowledge, Similarity, and Information (ProCKSI) implementing the protocol, published by Krasnogor and Pelta [24], and substantially extending it. This server facilitates protein structural comparison by allowing the user to compare multiple protein structures seamlessly using multiple similarity methods through a unique and integrated interface. As ProCKSI adheres to the philosophy mentioned above where different conceptions of similarities can be used under different circumstances, it deals well with comparisons of both very divergent structures and quite similar ones. Until now, methods were proposed that work well in either of these cases but not in both simultaneously. The first case is dealt with using the top level of the protocol, namely, the Universal Similarity Metric (USM, [45]) and the latter case by means of the Maximum Contact Map Overlap (MaxCMO) method $[20,21,23]$. As it has been shown in other contexts (e.g. protein structure prediction) that meta-servers sometimes outperform human experts $[46,47]$, the similarity results returned by these two methods can also be complemented with other comparison methods, and even integrated into a consensus similarity assessment. Hence, motivated by the observations above and in recognition that a) in many situations a very detailed comparison is needed and b) biologists may also want to compare 
a protein set from several viewpoints or conceptions of similarity simultaneously, ProCKSI harvests results from well-established external protein comparison servers and methods. It makes them available and readily comparable with one another, and combines the various similarity measures to give a consensus similarity profile for a given dataset.

The first level of similarity assessment utilises the USM as a similarity measure between two protein structures $s_{1}$ and $s_{2}$. Their contact map representations are then used to approximate heuristically the Kolmogorov complexity of the proteins, comparing their information content. The approximation of the Kolmogorov complexity is done using a compression algorithm (e.g. compress, gzip, bzip2, ppmz2). The pairwise similarities are then expressed as the Normalised Compression Distance (see Eq. $1)$, NCD, where $K\left(s_{i}\right)$ represents the Kolmogorov complexity of object $s_{i}$ and where $K\left(s_{i} \mid s_{j}\right)$ is the conditional complexity. NCD is a very effective universal, i.e. problem-domain independent, similarity metric particularly with distantly related structures [24] and sequences [48].

$$
\operatorname{NCD}\left(s_{1}, s_{2}\right)=\frac{\max \left\{K\left(s_{1} \mid s_{2}\right), K\left(s_{2} \mid s_{1}\right)\right\}}{\max \left\{K\left(s_{1}\right), K\left(s_{2}\right)\right\}}
$$

As the USM is a very general metric, for more fine grained comparisons ProCKSI implements a metaheuristic to compute the Maximum Contact Map Overlap (MaxCMO) of pairs of proteins counting the number of equivalent residues (alignments) and additionally the number of equivalent contacts (overlaps). Under the MaxCMO model, an amino acid residue $a_{1}$ from one protein is aligned to an amino acid residue $a_{2}$ from a second protein if a contact of $a_{1}$ in the first protein $\left(C\left(a_{1}\right)\right)$ can also be aligned to a contact of $a_{2}$ in the second protein $\left(C\left(a_{2}\right)\right)$ closing a cycle of size 4 in the graph representation of the contact map. A further restriction for the overlaps is that they should not produce crossing edges. That is, if $a_{1}$ is aligned to $a_{2}, C\left(a_{1}\right)$ is aligned to $C\left(a_{2}\right)$ and, without loss of generality, $a_{1}<C\left(a_{1}\right)$ (i.e. the atom or residue $a_{1}$ appears before than $C\left(a_{1}\right)$ in the sequence) then $a_{2}<C\left(a_{2}\right)$. Thus, an overlap in this model is a strong indication of topological similarity between the pair of proteins as it takes into consideration the local environment of each of the aligned residues. In addition to the two methods previously described, ProCKSI utilises the DaliLite workbench $[8,49]$ and the Combinatorial Extension (CE) method [11], both providing the statistical significance of an alignment (Z-score), the TM-align method [50] using TMscores, and the FAST method [28] providing $\mathrm{SN}$-scores as their key similarity measure.

The results are analysed with standard clustering methods. The clusters thus obtained can be visualised using either a linear, a circular or a hyperbolic representation of the hierarchical similarity tree [51] that captures the dataset's structural organisation. Additional analysis tools permit the comparison and integration of multiple similarity measures, so as to give a consensus similarity cluster. The analysis tools cannot only be used with ProCKSI's results but also in combination with additional similarity matrices that the user provides. Through this mechanism, the set of similarity matrices can be extended by any similarity measure that the user deems to be important allowing one to refine the similarity consensus for a given dataset. It allows the user to add different information which is not produced by the methods currently integrated with ProCKSI.

In addition to its core protocol for protein comparison as described above, ProCKSI aims to give an overall picture of the protein universe by providing, for each protein in the dataset, as much information and knowledge as possible. To this end, ProCKSI directly links to the PDB repository [52], the Structural Classification of Proteins (SCOP) database [53,54], and the Protein Structure Classification (CATH) database $[55,56]$. Sometimes, it might be useful not only to know more about the structure itself but also to get information about the literature where a certain protein occurs. ProCKSI therefore links to the information hyperlinked over proteins (iHOP) service $[57,58]$ providing an interactive network of proteins within the related literature.

In the next section we show the general architecture and workflow of ProCKSI and explain in detail the features introduced above.

\section{Implementation}

ProCKSI's workflow (Figure 1) consists of three main stages: Dataset Management, Calculation Management and Results Management. The latter includes the following parts: Overview Management, Structure Management, Analysis Management and the special Task Management that is associated with each of the different similarity comparison methods. In what follows we describe the functionality of each of these components and how they interoperate.

\section{Dataset Management}

The server can handle protein structure files in the Protein Data Bank (PDB) format, which can be downloaded directly from the PDB repository [52] by entering the PDB codes of the proteins. Alternatively, they can be uploaded from the user's local hard disc sequentially, i.e. one protein file at a time, or as archives (TAR, ZIP) containing multiple protein structure files. In either case, compressed files and archives in Z-, GZ-, or BZ2-format are also supported. The user can add further files, delete redundant 


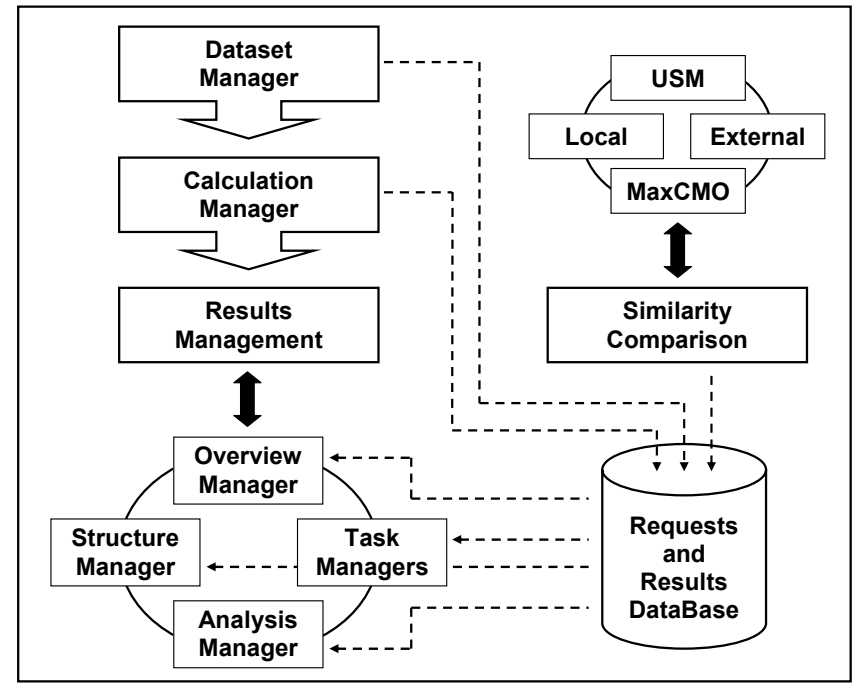

Figure I

ProCKSI's Architecture and Workflow. Overview over the workflow and architecture of ProCKSI with its multiple similarity comparison methods: Universal Similarity Metric (USM), Maximum Contact Map Overlap (MaxCMO), and other local and external methods. Currently, these are the DaliLite and TM-align methods, the Combinatorial Extension (CE) of the optimal path, and the FAST Align and Search Tool (FAST).

ones, and decompose structure files into all their models and chains. The user may select a subset of chains to be compared against each other, or perform an all-against-all chain comparison. If a PDB file was considered invalid, e.g. due to incomplete or ambiguous data within the file, the user has the opportunity to correct the errors before submitting the request for calculation. That is, the Dataset Management provides a flexible and user friendly interface for preparing the dataset for the further comparison in just a few steps.

\section{Calculation Management}

Once the protein files have been validated, the user must specify the calculation parameters, including the similarity methods to be used. Each comparison method requires specific parameters, which the Calculation Management allows to be set up. In the case of a similarity calculation with the USM method a USM equation [24,59] and a compression type must be chosen; for the MaxCMO method the number of restarts for the randomised solver has to be specified. The more restarts, the better the overlap values obtained, but this, of course, comes at the cost of compute time. All other methods take their standard parameters. When using the USM or MaxCMO method, each protein structure is then automatically converted into a contact map (Figure 2 - centre), based on a user-defined distance threshold and an exclusion window. The latter parameter specifies the number of nearest neighbour atoms in the sequence to be ignored while calculating the contact map.

As there is no general agreement on how to best represent a protein structure for comparison purposes, either $C_{\alpha}$ atoms [60-62] or $C_{\beta}$ atoms [63-66] can be chosen. The former representation focuses on the structure's backbone, whereas the latter one takes the residues' side chains into account. Alternatively and in contrast to other web servers (e.g. [67]), ProCKSI offers to calculate the residues' centres of mass in order to include both the backbone and side chain contributions at once.

\section{Results Management}

After a similarity comparison request has been submitted, it is added to a queue and the server returns a confirmation page that directly links to the Overview Manager. This gives a summary of all calculation parameters and the status, the submission, start and end times of all methods (tasks) that have been requested. As soon as all tasks have finished, the user receives a notification email and the expiration time (currently 7 days) for the entire request. Thereafter, the data are deleted.

ProCKSI returns a large variety of data and intermediate results that are handled through the Structures, Task and Analysis Management subsystems. These are described next.

\section{Structure Management}

As the USM and MaxCMO calculations both require contact maps as input, these are prepared before the actual similarity calculations take place. For each protein, all partial results are accessible for download and include a list of selected atoms, the protein's distance matrix and contact map, files with absolute and relative contacts and its contact vector (contact numbers). Thumbnails for the contact map of each protein are generated automatically, whereas high-quality pictures in different formats (PNG, PS, EPS), user-defined sizes and colours can be produced on demand. Two versions of contact map representations are available: dot matrix, and vertex \& edges. Additionally, the input protein structure can be displayed as plain text or as a static image preview [68]; an interactive VRML representation in $3 \mathrm{D}$ is also available and allows the user to explore and analyse the protein further (rotation, zoom, etc). The secondary structure is displayed as given in the PDB file (Figure 2 - left). For further and more detailed information about a protein, a direct link to the corresponding pages at the PDB repository [52], the SCOP $[53,54]$ and CATH $[55,56]$ structures classification databases, and the iHOP cross-literature database $[57,58]$ are also given. 

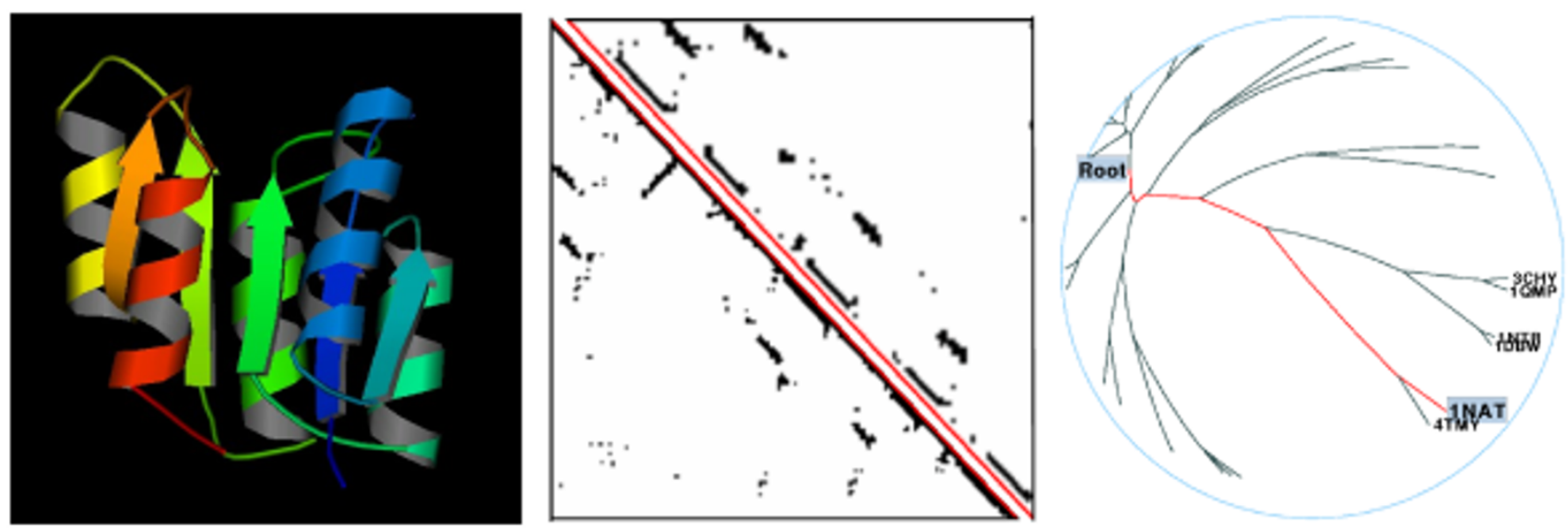

Figure 2

Building the Protein Universe. 3D proteins structures (left, [68]) can be represented as 2D contact maps that are used to compare pairs of proteins (centre) according to their USM and MaxCMO similarities. The resulting similarity matrices can be clustered in order to produce a hierarchical tree (right, [5I]). Spoof protein from Bacillus Subtilis (INAT) from the Skolnick dataset [23].

\section{Task Management}

As a request will usually involve several similarity methods, each of these is assigned to a separate Task Manager, which gives an overview of all similarity measures produced by any similarity method. These are Z-scores when using DaliLite or CE, overlap values in case of MaxCMO, whereas TM-align and FAST produce TM-scores and SNscores, respectively. Most methods provide RMSD values and the number of aligned residues, too. The USM method only returns the USM-score. Additionally, the user can access the natural output of the corresponding similarity method, e.g. the structural alignments or a list of structurally equivalent residue ranges.

\section{Analysis Management}

Once a task has finished, its similarity measures are available through the Analysis Management where they can be visualised and analysed. As the different similarity matrices are often either sensitive to protein size or do not have a fixed range of values, they must be normalised so that they can be used later to calculate a consensus similarity. Hence, besides providing the original similarity matrices (SM), ProCKSI also converts these into a standardised similarity matrix (SSM): each entry in the SSM matrix lies in the range $[0,1]$, with 0 describing the best (i.e. most similar), and 1 the worst (i.e. most dissimilar) similarity between two structures within the given set of proteins. The SSM matrix is then taken as input for clustering the protein set with one of a variety of hierarchical clustering methods, including e.g. the Unweighted Pair Group Method with Arithmetic mean (UPGMA) [69] or the Ward's Minimum Variance (WMV) method [70]. ProCKSI uses a local version of the Clustering Calculator [71] that outputs a plain text representation of the hierarchical tree and a file in the PHYLIP-format [72]. Especially useful for visualising large data sets, ProCKSI generates a HyperTree view [51] that opens as a Java applet from within the browser. This allows the user to display the protein set hierarchies as a circular, linear or as a hyperbolic tree. One benefit of using the hyperbolic representation is that it facilitates the navigation through the entire tree with a "fish-eye" perspective allowing to zoom in/out of regions of interest (Figure 2 - right). Not only can individual similarity measures be analysed as described before, but also the SSM can be combined in order to give a consensus picture of similarity. In turn, this consensus similarity matrix can be used as input for the clustering process thus obtaining a consensus hierarchical clustering tree.

\section{Results}

ProCKSI's core technologies, namely the USM and MaxCMO methods, and external servers and methods, namely the DaliLite, CE, FAST, and TM-align methods, have been introduced and evaluated independently in the past $[8,11,20,22-24,27,28,49,50]$. In this section though, we concentrate on ascertaining the added value of having, on the one hand, a unique interface to access all the previously mentioned methods, and on the other hand, the facility both to compare similarity assessments and to compute a consensus measure. We will present several case studies focusing on different aspects of ProCKSI's features and performance: a) the evaluation of some recent CASP results, introducing ProCKSI's new Consensus method, b) a new study reproducing the Hanks' and 
Hunt's classification scheme of protein kinases [73], which was originally derived from sequence comparisons, but this time verifying it using a consensus of different structural similarity methods, and c) an analysis of all methods implemented in ProCKSI using Receiver Operator Characteristics (ROC) with the Rost and Sander dataset. In addition to an analysis of the influence of different similarity methods and measures on the quality of the consensus, we conclude with benchmark tests measuring ProCKSI's total time needed to produce this similarity consensus.

\section{Evaluation of the CASP6 Results}

The evaluation of the CASP6 results is a challenging task, as it involves many protein structure files of a widely varying degree of similarity. Although protein scientists will often be interested in good alignments between pairs of closely related proteins, the capability of properly aligning distantly related structures is useful in ab-initio (new fold) structure prediction and the assessment of the predicted structures [74]. In CASP, the evaluators need to deal with literally tens of thousands of protein structure candidates that are often not too similar to the targets. The case of very different protein structures sometimes baffles structural alignment methods including LGA [12] that are regularly used to evaluate CASP results. ProCKSI, on the other hand, is likely to be less prone to producing a misleading ranking, because it harvests the results from various methods averaging them in order to produce a consensus.

For our experiments, we have chosen targets from both the CASP6 CM/easy and CM/hard category. The differentiation of targets into easy and hard is related to the degree of difficulty of predicting a model for the target using a given template, but does not reflect the evaluation process. For CM/easy targets, homologous structures can be found in databases, which might lead to many fairly good models. These can be very similar to each other and difficult to rank properly. On the other hand, the evaluation of the $C M / h a r d$ category is not straightforward either, as the structural similarity between model and target can be very low, due to the more difficult structural prediction task.

In the following, we discuss three examples (targets T0231, T0211 and T0196) that illustrate how ProCKSI deals with the difficulties described above. The targets were compared to all models proposed by the prediction servers that produced a native-like protein model including side chains. We used $\mathrm{C}_{\beta}$ atoms to represent the protein structure in this experiment in order to be consistent with the assessment of the CASP6 experiment [66] thus taking the positions of the side chains into account. ProCKSI's results from methods using contact maps, namely USM similarity, MaxCMO/Overlap and MaxCMO/Align values, and its new ProCKSI/Consensus method (using a total-evidence approach [47] combining all three taking the arithmetic average) are compared against GDT-TS, the main measure for structural similarity in CASP [75]. We provide the GDT-TS ranking results obtained from a sequence dependent analysis (SDA) as used in the official CASP evaluation procedure, and additionally from a sequence independent analysis (SIA), as MaxCMO's algorithm works in this mode.

Table 1 shows the ranking results of target T0231, a protein from the CM/easy category. This structure is the most conserved structure in CASP6 [76], i.e. homologous ones can be found in various databases, making its prediction simpler than it would otherwise be. The availability of several good, only slightly different models, makes the ranking process difficult as small differences between the candidate structures must be detected and evaluated. ProCKSI's results are in very good agreement with GDT-TS (SDA), the community's gold standard. At least three tar-

Table I: Evaluation of CASP Target T023 I. Comparison of the ranking results of target T023 I against 24 server predicted models, using different similarity methods. The GDT-TS results are obtained from calculations with sequence independent analysis (SIA) and sequence dependent analysis (SDA)

\begin{tabular}{|c|c|c|c|c|c|c|}
\hline Ranking & GDT-TS SDA & GDT-TS SIA & USM & MaxCMO Overlap & MaxCMO Align & $\begin{array}{l}\text { ProCKSI } \\
\text { Consensus }\end{array}$ \\
\hline I & TSO30 & TS289 & TS338 & TSO30 & TS338 & TS338 \\
\hline 2 & TS207 & TS519 & TSI86 & TSI39 & TS207 & TSO30 \\
\hline 3 & TS242 & TS283 & TS030 & TS338 & TS242 & TS207 \\
\hline 4 & TSI86 & TSI39 & TS207 & TS207 & TS033 & TSI86 \\
\hline 5 & TS338 & TS324 & TS400 & TS242 & TSI39 & TS242 \\
\hline 20 & TS45I & TS400 & TS45I & TS45I & TSII4 & TS45I \\
\hline 21 & TSIOI & TSII4 & TS38I & TSII4 & TS38I & TS38I \\
\hline 22 & TS304 & TSIOI & TSO19 & TS304 & TS304 & TS304 \\
\hline 23 & TS519 & TSOI9 & TS304 & TS519 & TS519 & TS519 \\
\hline 24 & TSOI9 & TS304 & TS519 & TSOI9 & TSOI9 & TSOI9 \\
\hline
\end{tabular}


Table 2: Evaluation of CASP Target T02 I I. Comparison of the ranking results of target T02 I I against 24 server predicted models, using different similarity methods. The GDT-TS results are obtained from calculations with sequence independent analysis (SIA) and sequence dependent analysis (SDA)

\begin{tabular}{|c|c|c|c|c|c|c|}
\hline Ranking & GDT-TS SDA & GDT-TS SIA & USM & MaxCMO Overlap & MaxCMO Align & $\begin{array}{c}\text { ProCKSI } \\
\text { Consensus }\end{array}$ \\
\hline I & TS45। & $\mathrm{TS} 213$ & TS45I & TS45I & TS45। & TS45I \\
\hline 2 & TS283 & TS38I & TS263 & TS283 & TS289 & TS283 \\
\hline 3 & TSIOI & TS324 & TSIOI & TS400 & TS283 & TS400 \\
\hline 4 & TS207 & TS290 & TS324 & TS38I & TS38I & TS38I \\
\hline 5 & TS375 & TS263 & TS207 & TS324 & TS324 & TS324 \\
\hline 20 & TS338 & TSOI9 & TSII4 & TS519 & TS304 & TS519 \\
\hline 21 & TS352 & TS033 & TS352 & TSI 86 & TS338 & TS338 \\
\hline 22 & TSI86 & TS352 & $\mathrm{TS} 033$ & TS338 & TS033 & TSO33 \\
\hline 23 & TS304 & TS186 & TSI86 & TS033 & TS352 & TS352 \\
\hline 24 & TS519 & TS519 & TS338 & TS352 & TSI 86 & TSI86 \\
\hline
\end{tabular}

gets ranked in the top five places by GDT-TS (SDA) can be found in similar places when using any of ProCKSI's similarity measures. More specifically, not only does ProCKSI find some major agreement in the most similar models for this target but also the three least similar models as ranked by MaxCMO/Overlap, MaxCMO/Align and ProCKSI/ Consensus match perfectly the ranking of GDT-TS (SDA).

Focusing on target T0211 of the CM/hard category [76] we find that GDT-TS (SDA) and ProCKSI/Consensus consider the same models for the first and second best models and that also the last six places, i.e. worst models, show considerable agreement (Table 2).

Interestingly, the results obtained by ProCKSI and GDTTS running in sequence independent mode differ more, when given targets T0231 and T0211, than when GDT-TS operates in sequence dependent mode. For example, considering T0231, we obtain no agreement between ProCKSI's top 5 models and GDT-TS (SIA) and only two (ProCKSI/Consensus) or three (MaxCMO) matches in the bottom 5 ranked models. These are quite surprising results as one would have expected the results of ProCKSI to match the sequence independent operation mode of GDT-TS better than the sequence dependent one, as ProCKSI does not use sequence information. Noting that CASP results are evaluated on the sequence dependent mode and that ProCKSI produced good agreement with it, we will investigate in the near future whether adding GDT-TS (SIA) to ProCKSI's pool of methods would be advantageous.

Using target T0196 as a third example taken from the CM/ hard category, we show that the combination of similarity criteria into a consensus can sometimes detect a better model than GDT-TS (SDA)(see Table 3 for details). Figure
3 illustrates the 3D structures of those models that are considered by the different methods to be the most similar to the target structure, called the "winner" of a certain method in the following. The ProCKSI/Consensus similarity measure detects a model that better resembles the overall structural features of T0196. More specifically, the candidate model selected by GDT-TS (SDA) has a fairly long segment of the chain that was not predicted correctly (blue), a helix structure was incorrectly suggested (orange) and the $\beta$ sheets are in the wrong places (green). This model is ranked last by ProCKSI/Consensus.

Next, we analysed the sequence similarities between the target structure and the winner of each method. Using the MaxCMO method to produce all sequence alignments, we obtained up to $76.7 \%$ (target vs. winner of GDT-TS), up to 98.6\% (target vs. winner of MaxCMO/Overlap) and up to 95.8\% (target vs. winner of USM and ProCKSI/Consensus) correctly aligned residues, having taken the best results of multiple MaxCMO runs. This illustrates that the MaxCMO method does not only detect the most similar model according to its overlap values, but also gives the better alignment with the highest sequence similarity. When using GDT-TS in sequence independent mode instead, both methods suggest the same model for the best structural match and even agree with almost all models within the top five in the ranking.

Summing up, we found a very good agreement between ProCKSI/Consensus and CASP's GDT-TS method, although the two run in different modes: the former obtains its results from sequence-independent calculations while the latter additionally uses sequence information. When both methods suggest a different winner in their rankings, the ProCKSI/Consensus method can detect a 
Table 3: Evaluation of CASP Target T0 I 96. Comparison of the ranking results of target T0 I 96 against 22 server predicted models, using different similarity methods. GDT-TS results are obtained from calculations with sequence independent analysis (SIA) and sequence dependent analysis (SDA)

\begin{tabular}{|c|c|c|c|c|c|c|}
\hline Ranking & GDT-TS SDA & GDT-TS SIA & USM & MaxCMO Overlap & MaxCMO Align & $\begin{array}{l}\text { ProCKSI } \\
\text { Consensus }\end{array}$ \\
\hline I & TS223 & TS352 & TS352 & TS030 & TS352 & TS352 \\
\hline 2 & TS213 & TS290 & TS030 & TS38I & TS030 & TS030 \\
\hline 3 & TS45I & TS38I & TS400 & TS352 & TS38I & TS38I \\
\hline 4 & TS033 & TS030 & TSO30 & TS400 & TS290 & TS290 \\
\hline 5 & TS376 & TS223 & TS400 & TS304 & TS324 & TS324 \\
\hline 18 & TS338 & TS338 & TS289 & TS338 & TS304 & TS338 \\
\hline 19 & TS324 & TS242 & TS376 & TS283 & TSII3 & TSII3 \\
\hline 20 & TS304 & TSO33 & TSII3 & TSII3 & TS283 & TS283 \\
\hline 21 & TS289 & TS207 & TS213 & $\mathrm{TS} 213$ & $\mathrm{TS} 213$ & $\mathrm{TS} 213$ \\
\hline 22 & TS352 & TS375 & TS223 & TS223 & TS223 & TS223 \\
\hline
\end{tabular}

better model with a higher similarity (value) and even a higher sequence similarity.

\section{Structural Comparison and Clustering of Protein Kinases}

In the following, we perform an additional (and more detailed) analysis of ProCKSI's functionality by concentrating on a set of protein kinases (PK). These are then compared and clustered according to their structural similarity. These structure-only based results are then compared with the original classification scheme by Hanks and Hunter $(\mathrm{HH})[73]$ that was based on sequence similarity. As was the case for the CASP targets, ProCKSI's integration of several structural similarity criteria allows it to reproduce the original classification without using sequence information.

Kinases are proteins that catalyse the transfer of a phosphate to a protein substrate and form a reversible equilibrium with phosphatases as their counterpart [77]. They

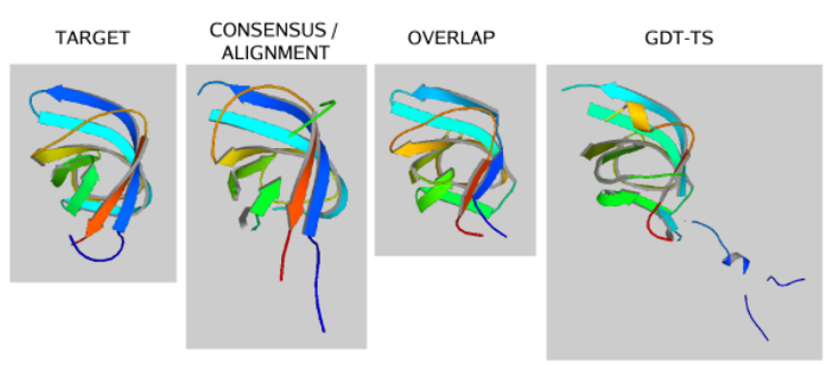

Figure 3

Evaluation of Models against CASP Target T0 I 96. CASP target T0196 was compared against all submitted server models using ProCKSI's similarity methods USM, Max$\mathrm{CMO}$, and its new Consensus method. The most similar structures to the target detected by each method are displayed next to each other. - The protein structure pictures were generated by ProCKSI using MolScript [68]. comprise a huge group of enzymes that play an essential role in most of the major cellular processes such as cellular differentiation and repair, cell proliferation, etc [78]. In an attempt to organise the set of protein kinases, Hanks and Hunter [73] classified them accordingly to their sequence into 5 broad groups (super-families), 44 families, and 51 domains (sub-families). Several additions and refinements to this classification (e.g. [79-82]) were later introduced.

\section{Dataset Preparation}

As the dataset for our experiments, we have chosen the structures published on a mirror site [83] of the Protein Kinase Resource (PKR) web site $[77,78,84]$, which is an online compendium for information on protein kinases [77]. We use Hanks' and Hunter's original classification as it goes hand in hand with this dataset that comprises 46 structures from 9 different groups (super-families), namely 1) cAMP Dependent Kinases, 2) Protein Kinases C, 3) Phosphorylase Kinases, 4) Calmodulin Kinases, 5) Casein Kinases, 6) Cyclin Dependent Kinases, 7) Tyrosine Kinases, 8) Mitogen Activated Kinases, and 9) Twitchen Kinases. For each protein, we obtained detailed information about its class, fold, superfamily, family, protein, and species from the SCOP database, release 1.69 [85], which is summarised in Table 4. It should be mentioned that Hanks' and Hunter's original classification scheme showed separate super-families for Tyrosine Kinases (TK) and Serine/Threonin Kinases (S/TK). Starting with SCOP release 1.65, these have been combined into one single family comprising all protein kinases with a characteristic catalytic subunit [54]. In this text, we refer to the new classification, but for the sake of completeness, the old classification is denoted in parentheses.

A further first analysis of the dataset revealed that protein 1 RGS, a double stranded $\beta$-helix, was given as the only all beta protein within an alpha+beta class (HH cluster 1), and 
Table 4: Protein Kinase Dataset. Detailed SCOP classification for each protein domain in the Protein Kinase (PK) dataset, grouped according to Hanks' and Hunter's (HH) original classification scheme

\begin{tabular}{|c|c|c|c|c|c|c|c|c|}
\hline \multirow[t]{2}{*}{ HH Cluster } & \multirow[t]{2}{*}{ SubCluster } & \multirow[t]{2}{*}{ ProteinDomain } & \multicolumn{6}{|c|}{ SCOP Classification Level } \\
\hline & & & Class & Fold & Superfamily & Family & Domain & Species \\
\hline \multirow[t]{12}{*}{1} & $A$ & dlapme_ & $\alpha+\beta$ & PK-like & PK-like & PK c.s. (S/TK) & cAMP-dep. PK, c.s. & mouse \\
\hline & & dlatpe_ & $\alpha+\beta$ & PK-like & PK-like & PK c.s. (S/TK) & cAMP-dep. PK, c.s. & mouse \\
\hline & & dlbkxa_ & $\alpha+\beta$ & PK-like & PK-like & PK c.s. (S/TK) & cAMP-dep. PK, c.s. & mouse \\
\hline & & dlfmoe & $\alpha+\beta$ & PK-like & PK-like & PK c.s. (S/TK) & cAMP-dep. PK, c.s. & mouse \\
\hline & & d2cpke_- & $\alpha+\beta$ & PK-like & PK-like & PK c.s. (S/TK) & cAMP-dep. PK, c.s. & mouse \\
\hline & B & dlcdka & $\alpha+\beta$ & PK-like & PK-like & PK c.s. (S/TK) & cAMP-dep. PK, c.s. & pig \\
\hline & & dlcmke_ & $\alpha+\beta$ & PK-like & PK-like & PK c.s. (S/TK) & cAMP-dep. PK, c.s. & pig \\
\hline & & dlctpe_ & $\alpha+\beta$ & PK-like & PK-like & PK c.s. (S/TK) & cAMP-dep. PK, c.s. & pig \\
\hline & C & dlstce_ & $\alpha+\beta$ & PK-like & PK-like & PK c.s. (S/TK) & cAMP-dep. PK, c.s. & cow \\
\hline & & dlydre_- & $\alpha+\beta$ & PK-like & PK-like & PK c.s. (S/TK) & cAMP-dep. PK, c.s. & cow \\
\hline & & dlydse_ & $\alpha+\beta$ & PK-like & PK-like & PK c.s. (S/TK) & cAMP-dep. PK, c.s. & cow \\
\hline & & dlydte_- & $\alpha+\beta$ & PK-like & PK-like & PK c.s. (S/TK) & cAMP-dep. PK, c.s. & cow \\
\hline \multirow[t]{2}{*}{2} & - & diptq__ & small & PK c.-r. domain & PK c.-r. domain & PK c.-r. domain & PK C-delta (PKCdelta) & mouse \\
\hline & & dlptr_ & small & PK c.-r. domain & PK c.-r. domain & PK c.-r. domain & PK C-delta (PKCdelta) & mouse \\
\hline 3 & - & dlphk_ & $\alpha+\beta$ & PK-like & PK-like & PK c.s. (S/TK) & $\gamma$-subunit glycogen Phk & rabbit \\
\hline \multirow[t]{4}{*}{4} & A & $\mathrm{dla} 06$ & $\alpha+\beta$ & PK-like & PK-like & PK c.s. (S/TK) & Calmodulin-dep. PK & rat \\
\hline & B & dlcdma & $\alpha$ & EF Hand-like & EF-hand & Calmodulin-like & Calmodulin & cow \\
\hline & & $\mathrm{dlcmla}_{-}$ & $\alpha$ & EF Hand-like & EF-hand & Calmodulin-like & Calmodulin & cow \\
\hline & & $\mathrm{dlcm} 4 \mathrm{a}_{-}$ & $\alpha$ & EF Hand-like & EF-hand & Calmodulin-like & Calmodulin & cow \\
\hline \multirow[t]{3}{*}{5} & A & dIIr4a & $\alpha+\beta$ & PK-like & PK-like & PK c.s. (S/TK) & Casein kinase-2, CK2 & maize \\
\hline & B & dlcsn & $\alpha+\beta$ & PK-like & PK-like & PK c.s. (S/TK) & Casein kinase-I, CKI & fission yeast \\
\hline & & $\mathrm{d} 2 \mathrm{csn}$ & $\alpha+\beta$ & PK-like & PK-like & PK c.s. (S/TK) & Casein kinase-I, CKI & fission yeast \\
\hline \multirow[t]{5}{*}{6} & - & dlaql__ & $\alpha+\beta$ & PK-like & PK-like & PK c.s. (S/TK) & Cyclin-dep. PK, CDK2 & human \\
\hline & & dlfina & $\alpha+\beta$ & PK-like & PK-like & PK c.s. (S/TK) & Cyclin-dep. PK, CDK2 & human \\
\hline & & dlhck & $\alpha+\beta$ & PK-like & PK-like & PK c.s. (S/TK) & Cyclin-dep. PK, CDK2 & human \\
\hline & & dlhcl__ & $\alpha+\beta$ & PK-like & PK-like & PK c.s. (S/TK) & Cyclin-dep. PK, CDK2 & human \\
\hline & & dljsua_ & $\alpha+\beta$ & PK-like & PK-like & PK c.s. (S/TK) & Cyclin-dep. PK, CDK2 & human \\
\hline 7 & A & dlad5al & $\beta$ & SH3-like barrel & SH3-domain & SH3-domain & Hemapoetic cell kinase Hck & human \\
\hline
\end{tabular}


Table 4: Protein Kinase Dataset. Detailed SCOP classification for each protein domain in the Protein Kinase (PK) dataset, grouped according to Hanks' and Hunter's (HH) original classification scheme (Continued)

\begin{tabular}{|c|c|c|c|c|c|c|c|c|}
\hline & & dlad5a2 & $\alpha+\beta$ & SH2-like & SH2 domain & SH2 domain & Hemopoetic cell kinase Hck & human \\
\hline & & dlad5a3 & $\alpha+\beta$ & PK-like & PK-like & PK c.s. (TK) & Hemopoetic cell kinase Hck & human \\
\hline & & dlfmk_l & $\beta$ & SH3-like barrel & SH3-domain & SH3-domain & c-src protein TK & human \\
\hline & & dlfmk_2 & $\alpha+\beta$ & SH2-like & $\mathrm{SH} 2$ domain & $\mathrm{SH} 2$ domain & c-src TK & human \\
\hline & & dlfmk_3 & $\alpha+\beta$ & PK-like & PK-like & PK c.s. (TK) & c-src TK & human \\
\hline & & d2hckal & $\beta$ & SH3-like barrel & SH3-domain & $\mathrm{SH} 3$-domain & Hemapoetic cell kinase Hck & human \\
\hline & & d2hcka2 & $\alpha+\beta$ & SH2-like & $\mathrm{SH} 2$ domain & $\mathrm{SH} 2$ domain & Hemopoetic cell kinase Hck & human \\
\hline & & $\mathrm{d} 2 \mathrm{hcka} 3$ & $\alpha+\beta$ & PK-like & PK-like & PK c.s. (TK) & Haemopoetic cell kinase Hck & human \\
\hline & & d2ptk_l & $\beta$ & SH3-like barrel & SH3-domain & SH3-domain & c-src protein TK & chicken \\
\hline & & $\mathrm{d} 2 \mathrm{ptk} \_2$ & $\alpha+\beta$ & SH2-like & $\mathrm{SH} 2$ domain & $\mathrm{SH} 2$ domain & c-src TK & chicken \\
\hline & & d2ptk_3 & $\alpha+\beta$ & PK-like & PK-like & PK c.s. (TK) & c-src TK & chicken \\
\hline & B & dlaotf_ & $\alpha+\beta$ & SH2-like & $\mathrm{SH} 2$ domain & $\mathrm{SH} 2$ domain & TK Fyn & human \\
\hline & & dlblj_ & $\alpha+\beta$ & SH2-like & $\mathrm{SH} 2$ domain & $\mathrm{SH} 2$ domain & P55 Blk protein TK & mouse \\
\hline & & dlcsya_ & $\alpha+\beta$ & SH2-like & $\mathrm{SH} 2$ domain & $\mathrm{SH} 2$ domain & Syk TK & human \\
\hline & & dlcwea & $\alpha+\beta$ & SH2-like & $\mathrm{SH} 2$ domain & $\mathrm{SH} 2$ domain & p56-lck TK & human \\
\hline & C & dlfgka & $\alpha+\beta$ & PK-like & PK-like & PK c.s. (TK) & $\begin{array}{c}\text { Fibroblast growth factor receptor } \\
\text { | }\end{array}$ & human \\
\hline & & dlir3a & $\alpha+\beta$ & PK-like & PK-like & PK c.s. (TK) & Insulin receptor & human \\
\hline & & dlirk & $\alpha+\beta$ & PK-like & PK-like & PK c.s. (TK) & Insulin receptor & human \\
\hline & & d3lck _ & $\alpha+\beta$ & PK-like & PK-like & PK c.s. (TK) & Lymphocyte kinase (Ick) & human \\
\hline 8 & A & dlerk & $\alpha+\beta$ & PK-like & PK-like & PK c.s. (S/TK) & MAP kinase Erk2 & rat \\
\hline & B & dlian & $\alpha+\beta$ & PK-like & PK-like & PK c.s. (S/TK) & MAP kinase p38 & human \\
\hline & & dlp38_ & $\alpha+\beta$ & PK-like & PK-like & PK c.s. (S/TK) & MAP kinase p38 & mouse \\
\hline & & diwfe & $\alpha+\beta$ & PK-like & PK-like & PK c.s. (S/TK) & MAP kinase p38 & human \\
\hline 9 & A & dlkoa_l & $\beta$ & IG-like $\beta$-sandwich & IG & I set domains & Twitchin & nematode \\
\hline & & dlkoa_2 & $\alpha+\beta$ & PK-like & PK-like & PK c.s. (S/TK) & Twitchin, kinase domain & $\begin{array}{l}\text { Caenorhabditi } \\
\text { s elegans }\end{array}$ \\
\hline & B & dlkoba & $\alpha+\beta$ & PK-like & PK-like & PK c.s. (S/TK) & Twitchin, kinase domain & $\begin{array}{l}\text { California sea } \\
\text { hare }\end{array}$ \\
\hline
\end{tabular}

IG = Immunoglobulin, PhK = Tyrosine Phosphorylase Kinase, S/TK = Serine/Threonin Kinase, $\mathrm{TK}=$ Tyrosine Kinase, c.s. = catalytic subunit, c.-r. $=$ cysteine-rich., dep. $=$ dependent 
was therefore removed from the dataset. A further, more detailed analysis of the remaining 45 structures revealed a more fine-grained similarity structure than that suggested by Hanks and Hunter. In most of the clusters given in Table 4, the protein kinases can be sub-divided into subclusters with common features. This could be either a common class (e.g. cluster 4), a common fold (e.g. cluster 7 ), or even a common species (e.g. cluster 1 ). The members of clusters 2, 3 and 6 cannot be further differentiated as they share the same features up to the species level, respectively. In order to capture this intrinsic similarity structure, we have sub-divided the HH clusters according to the biggest set of common features, labelling these with letters in addition to the original cluster number. Clusters 2, 3 and 6 are not considered as they cannot be further sub-divided. To illustrate this, consider proteins 1AD5 and 1FGK, both belonging to HH cluster 7 (Tyrosine Kinases). The former is built up from multiple domains while the latter has just one domain. These are therefore put into two different sub-clusters, 7A and 7C.

\section{Kinase Structural Classification Results}

From the available methods in ProCKSI (USM, MaxCMO, DaliLite, CE, TM-align, FAST), we have performed similarity calculations between all the 45 Kinases using the USM, the MaxCMO and the DaliLite methods. These 45 proteins imply at least 1035 pairwise comparisons per parameter setting of each algorithm. These include the comparison of a structure with itself, as the self-similarity values are needed to standardise the final similarity matrix. On top of each request using one of three different contact map thresholds, seven different clustering methods can be applied. ProCKSI's interface handles the set up of all these comparisons in an automatic and user-friendly way.

In particular, $\mathrm{C}_{\alpha}$ atoms were chosen to represent the protein structures with all methods, taking into account that DaliLite uses them by default. Distance thresholds of 5.0 $\AA, 7.5 \AA$ and $10.0 \AA$ were chosen in order to produce the contact maps necessary for USM and MaxCMO. Rather than prescribing a given clustering method, following ProCKSI's philosophy, our decision support system allows the user to choose, and seamlessly try, different clustering algorithms on his/her datasets. In this example the similarity matrices obtained from USM, MaxCMO and DaliLite were standardised and fed into each of the clustering methods available in ProCKSI. For brevity we report only the results using the $7.5 \AA$ and the WMV clustering algorithm.

The MaxCMO method distinguishes the kinases in our dataset clearly upon the class/fold level and separates them into two clusters (Figure 4 - left): alpha+beta/PK-like proteins and others. The latter comprise all small proteins
(2), all alpha proteins with EF Hand-like fold (4B), and such from the alpha+beta class but with SH2-like fold (7B), all of them being identified and clustered correctly. One could assume that multidomain proteins with diverse classes/folds (7A; 1KOA) should be found in this cluster, too, but a more in-depth analysis revealed that the domains of these proteins show mainly alpha+beta/PKlike fold ( $>62.5 \%$ in the entire structure). Consequently, they are correctly grouped together with proteins resembling the same class/fold properties. As this cluster is detected by each similarity measure almost always correctly, it is highlighted with a green box in all dendrograms (Figures 4 and 5). While DaliLite wrongly adds a fairly different protein to this cluster (1IAN from HH cluster 8 ), the USM method just reverses the order of the penultimate and the last clustering step. In addition, both the USM and DaliLite/Z measures are able to detect similarities up to the species level (Figure 4 - middle). Consider, for instance, HH cluster 1 (blue box) containing similar kinases from mice, pigs and cows, which are clustered comparably well (errors indicated in blue within the blue cluster). Both methods also produce a "mixed bag" of proteins (red box) that are less similar to all others outside the green cluster, with the USM method misplacing 1AQ1 (6) into the red cluster.

The results illustrate both the strengths and minor flaws of different similarity methods taken as independent criteria of similarity. We proceed next to analyse the results of the Kinase structural classification when taking these criteria in combination.

The previous analysis shows that including similarity matrices derived from alignment numbers (MaxCMO/ Align, DaliLite/Align) always cluster proteins within the green box correctly, but partially destroy the good clustering of the other proteins. Thus, they are not considered as candidates for producing a high-quality consensus clustering for this dataset. The best result was obtained combining the USM and DaliLite/Z similarity measures, which reproduced the red, blue and green clusters correctly (Figure 5 - left). Both the DaliLite's error within the green cluster and the USM error within the red cluster were corrected while the proteins in the blue cluster were correctly classified. Surprisingly enough, adding the MaxCMO/ Overlap similarity measure, which was only able to produce the correct clustering within the green cluster, still gives a comparably good result (Figure 5 - right).

Taken together, these results show that the combination of a range of algorithms that employ different similarity criteria has the potential to overcome the inherent weaknesses in each one of them, and thus is able to produce a robust more similarity result. 

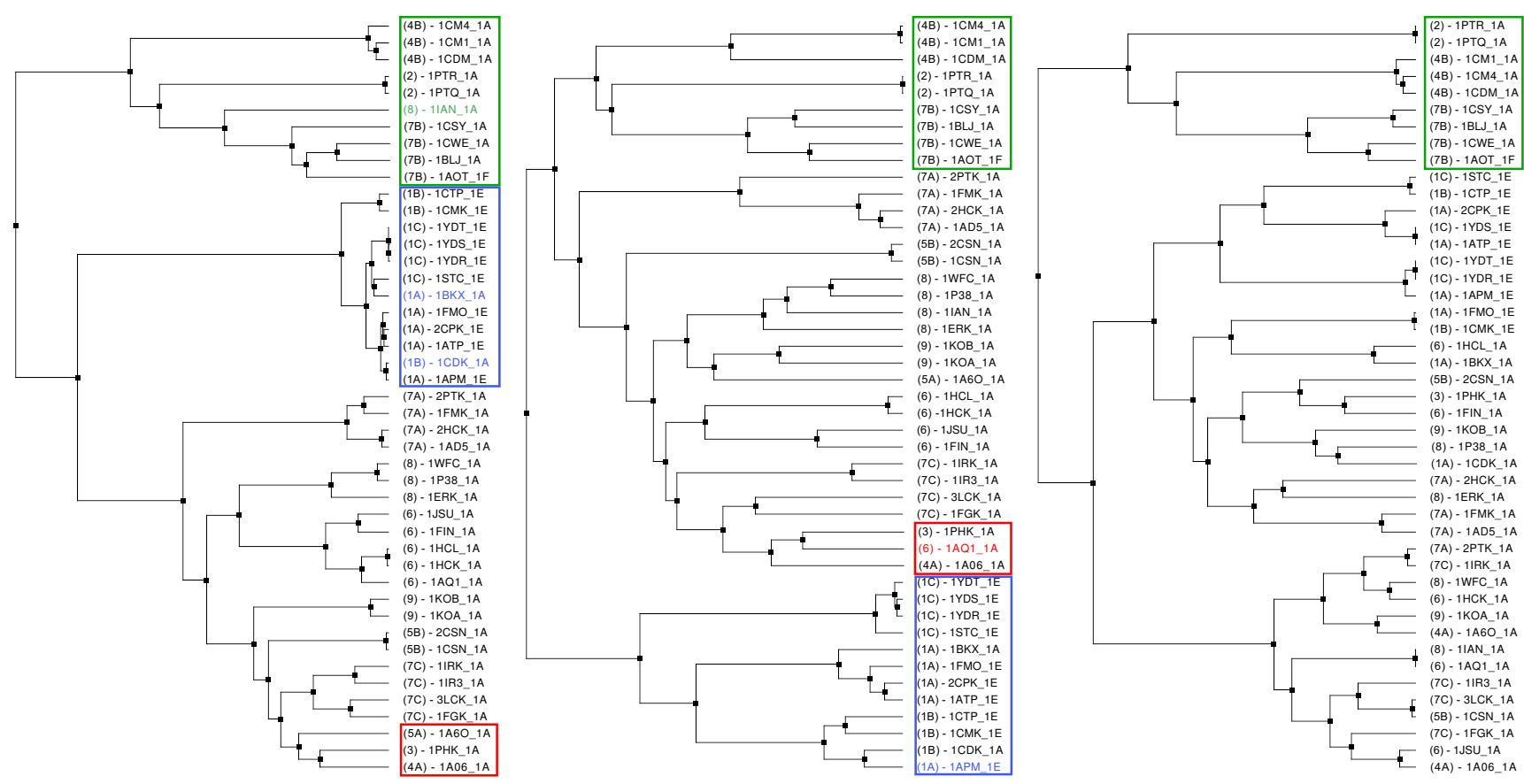

\section{Figure 4}

Clustering the Kinase Dataset using Single Similarity Measures. The Kinase dataset was clustered with the Ward's Minimum Variance (WMV) method in conjunction with the MaxCMO/Overlap (left), the USM (middle), and the DaliLite/Z (right) similarity measures. The meaning of the different coloured boxes is explained in the text in detail. - The hierarchical tree images were generated by ProCKSI using HyperTree [5I].

\section{Evaluation of Multiple Similarity Comparison Methods using ROC Curves}

In the previous section, we investigated in detail by manual inspection how the similarity comparison methods USM, MaxCMO and DaliLite can be combined in order to achieve an optimal consensus result. In this section, we take the next step towards a fully-automated decision support system by analysing the quality and performance of the six different similarity comparison methods currently included in ProCKSI by means of Receiver Operator Characteristics (ROC) [86]. These are USM, MaxCMO, DaliLite, CE, TM-align, and FAST, providing a total number of 15 similarity measures (compare section Task Management for details).

In the following, we describe the experimental setup, explain how ROC curves are generated, and employ this technique to determine the most promising methods to include in order to produce an even better consensus method.

\section{Dataset and Gold Standard}

For our analysis, we have chosen the Rost and Sander dataset (RS126), which was designed for the secondary structure prediction of proteins with a pairwise sequence similarity of less than $25 \%$ [87]. Here, we not only compare the proteins' secondary structures, but analyse the performance of ProCKSI's similarity comparison methods according to the proteins' classification as given by SCOP, release 1.69 [85]. We adopted this manually curated database as our gold standard containing expert knowledge for each of its hierarchical classification levels: Class, Fold, Superfamily, Family, Protein, and Species.

The dataset itself consists of 126 globular proteins, 18 of them with more than one domain. In order to allow the comparison of entire chains instead of breaking down the protein into domains, we have merged and re-classified all of a protein's multiple domains. In contrast to other over-simplified approaches like [88], where all multidomain proteins were merged with SCOP's already existing "multi-domain" class, we tried to preserve as much information as possible on each hierarchy level. If two domains disagreed in all classification levels, we also merged and re-classified them as "multi-domain". Moreover, moving down the hierarchy from the Class to the Species level, we kept the original classification for those levels that matched. For instance, if the class of two domains was given as "all alpha", but they showed different classifications for all other levels, then the class of the 


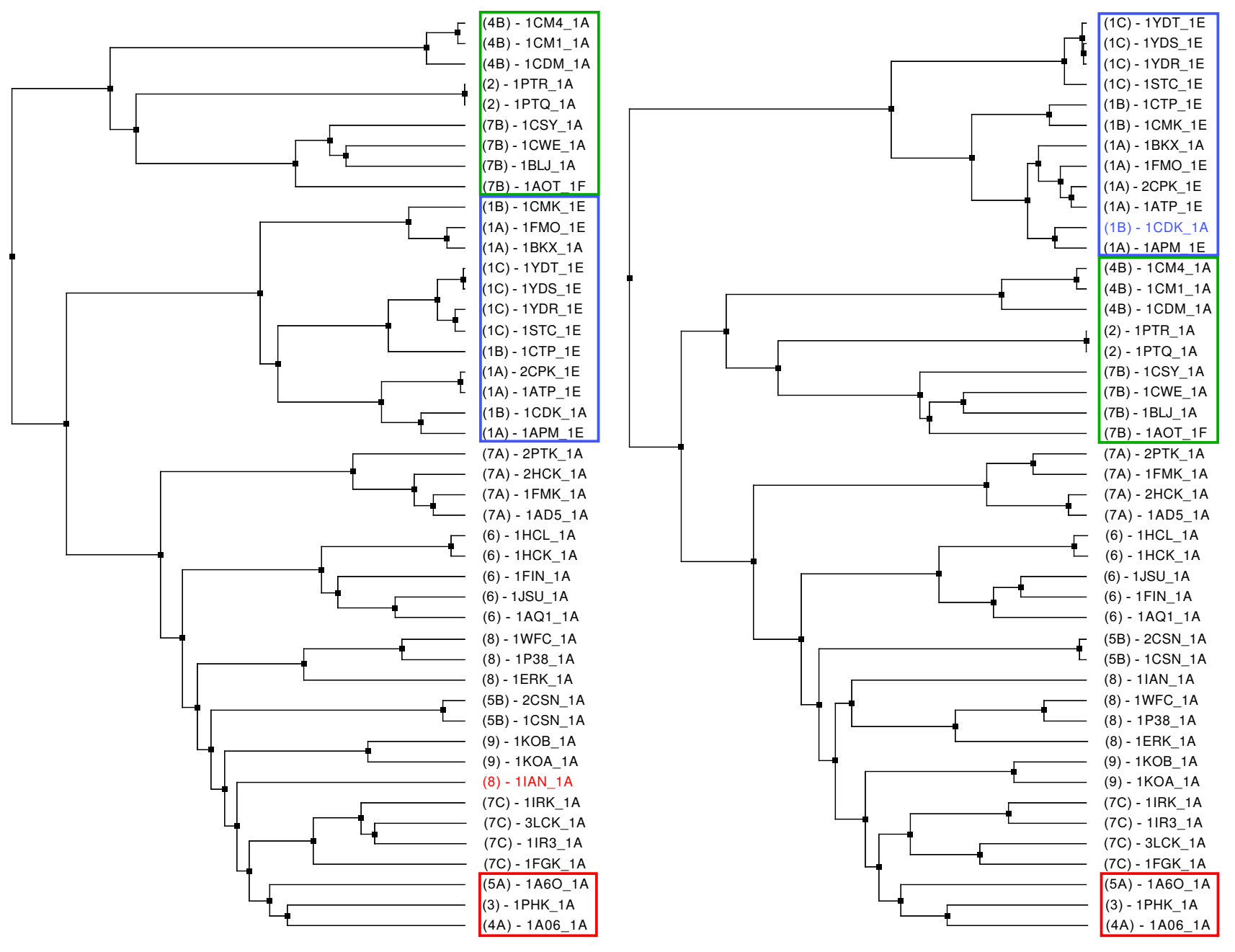

\section{Figure 5}

Clustering the Kinase Dataset using Consensus Similarity Measures. The Kinase dataset was clustered with the Ward's Minimum Variance (WMV) method in conjunction with a consensus similarity measure of the USM and DaliLite/Z (left), and the USM, DaliLite/Z, and MaxCMO/Overlap (right) similarity measures. The meaning of the different coloured boxes is explained in the text in detail. - The hierarchical tree images were generated by ProCKSI using HyperTree [5I].

entire chain was kept as "all alpha", but all other levels were re-classified as "multi-domain". Thus, in contrast to the approach of [88], comparing this chain with another "all alpha" chain counts as a correct classification (true positive) in the ROC analysis (see below).

\section{Introduction to ROC Analysis}

ROC analyses have been widely employed, e.g. in signal detection theory [89], machine learning [90], and diagnostic testing in medicine [91]. Recently, they have also been used for the evaluation of structural similarity and alignment methods $[88,92,93]$.

The performance of such a comparison or alignment method is measured by its ability to predict the degree of similarity between pairs of proteins, and to produce a relative ranking of similar (positive) and dissimilar (negative) pairs. The fraction of correctly classified positives (true positives) and the number of wrongly classified positives (false positives) in relation to the real number of positives gives the true positive rate (TPr) and false positive rate $(\mathrm{FPr})$, respectively. In a ROC graph, TPr and FPr are plotted against each other using a continuously varying decision threshold discriminating between true and false positives. The diagonal line between $(0,0)$ and $(1,1)$ denotes classifiers without any predictive power as they produce the correct classification just by chance [88]. The further a ROC curve is to the north-west in the graph, the better is the classifier, whereas classifiers in the south-east 
region have strong predictive power, but lead to wrong (opposite) conclusions [86].

In order to compare the complex performance of different similarity comparison methods, a ROC curve can be reduced to a single, scalar measure given as the Area Under the Curve (AUC). As the best method will have the uppermost (north-western) curve, it will have the largest AUC value, whereas an AUC value below 0.5 indicates an incorrect prediction.

\section{Comparison of Methods using ROC curves}

For each classification level, we generated ROC graphs using all available similarity measures, and combined all of them in order to produce a consensus (Consensus/All). Figure 6 shows the ROC graph for the Class level as a representative in order to explain some details of the graphs further: In this graph, all DaliLite measures display an unusual straight line from an FP rate of about 0.2 onwards. This artifact emerges from the fact that DaliLite does not return any similarity values for pairs of proteins with very low similarity. As a consequence, all those pairs are assigned the worst possible value (1.0) in the standardised similarity matrix and cannot be ranked unambiguously for the generation of the ROC curves. Not having more information at hand to predict the method's expected performance, we do not calculate any ROC points for any but the first of subsequent pairs with the same similarity value [86]. We obtain a straight line between the latter and the $(1,1)$ point, which is always present.

In the following, we analyse the performance of all included similarity measures using AUC values (Table 5 ). We found consistently in all hierarchy levels that RMSD values do not seem to be good similarity measures, giving very low AUC values, even below 0.5 . On the other hand, it is DaliLite/RMSD that could be used as a good similarity predictor ranking within the best four methods in almost all hierarchy levels except the Class level. Besides, the latter is the only level, where both FAST/Align and FAST/SN rank within the best three methods. In all other levels, CE/ Z, DaliLite/Z, and DaliLite/Align show the best performance.

Next, we analysed if the combination of different similarity measures would improve the results using just one single measure. As expected, indiscriminate averaging of all available measures (Consensus/All, Table 5) gave worse results than the best measure in each hierarchy level, since RMSD values were included, shifting the average to lower values. Selecting the best measure of each similarity comparison method (e.g. CE/Z, DaliLite/Z, FAST/SN, MaxCMO/Overlap, TM-align/TM, and USM/USM in the Fold level) led to an overall improved performance for all but
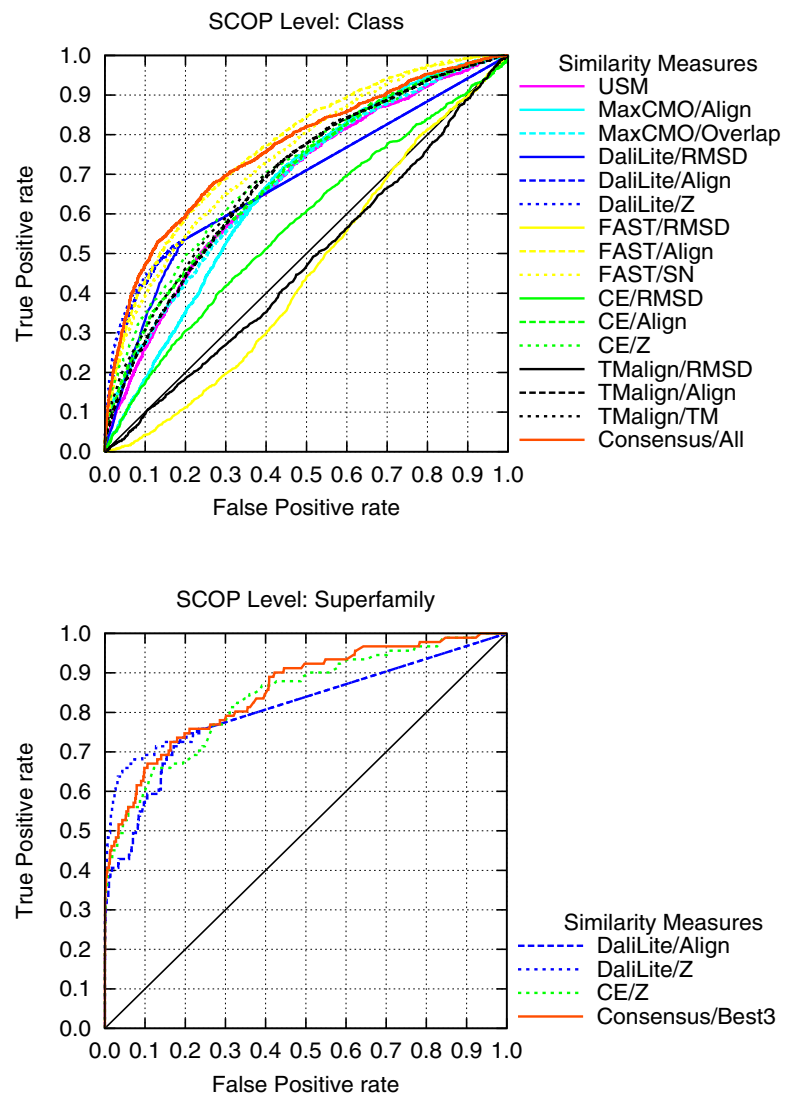

\section{Figure 6}

ROC Analysis for the Rost and Sander Dataset. ROC analysis for all available similarity comparison methods in ProCKSI for the Rost and Sander dataset using SCOP's Class level as gold standard (top). ROC analysis showing the better performance of ProCKSI's Consensus/Best3 method compared to each contributing single one using SCOP's Superfamily level as gold standard (bottom).

the Class levels. A further reduction of the contributing methods, selecting the three best ones, showed an unexpected result. In all but the Superfamily and the Protein levels, the performance of the Consensus/Best 3 method was better, having a greater AUC value than any of the single methods (compare Figure 6). The same synergistic effect was achieved for the two exceptions mentioned by forming the consensus from the two methods with the highest AUC values. The reason for this synergistic effect lies in an improved ranking of the pairs of proteins, having obtained similarity values that better discriminate between "similar" and "dissimilar".

These synergistic effects prove that ProCKSI's new Consensus measure can outperform even well established and reliable similarity comparison measures like DaliLite and $\mathrm{CE}$. Hence, in order to maximise this synergy, finding the 
Table 5: ROC Analysis for the Rost and Sander Dataset. Analysis of the performance of different similarity measures in terms of AUC values for each SCOP classification level using the Rost and Sander dataset. The Consensus measures are composed of $a \mathrm{CE} / Z$, DaliLite/Z, FAST/Align, MaxCMO/Overlap, TM-align/TM, and USM/USM for the Class and Species level, and CE/Z, DaliLite/Z, FAST/ SN, MaxCMO/Overlap, TM-align/TM, and USM/USM for all remaining levels, ${ }^{b}$ FAST/Align, FAST/SN, and DaliLite/Z for the Class level, and CE/Z, Dali/Z, and Dali/Align for all remaining levels, and c FAST/Align and FAST/SN for the Class level, and CE/Z and Dali/Z for all remaining levels.

\begin{tabular}{|c|c|c|c|c|c|c|c|}
\hline \multirow[t]{2}{*}{ Method } & \multirow[t]{2}{*}{ Measure } & \multicolumn{6}{|c|}{ AUC Values for SCOP Classification Level } \\
\hline & & Class & Fold & Superfamily & Family & Protein & Species \\
\hline \multirow[t]{3}{*}{ CE } & RMSD & 0.574 & 0.776 & 0.754 & 0.718 & 0.625 & 0.626 \\
\hline & Align & 0.694 & 0.704 & 0.660 & 0.643 & 0.503 & 0.486 \\
\hline & $\mathrm{z}$ & 0.712 & 0.848 & 0.838 & 0.826 & 0.769 & 0.759 \\
\hline \multirow[t]{3}{*}{ DaliLite } & RMSD & 0.677 & 0.807 & 0.794 & 0.786 & 0.746 & 0.751 \\
\hline & Align & 0.693 & 0.827 & 0.807 & 0.792 & 0.755 & 0.759 \\
\hline & $\mathrm{Z}$ & 0.696 & 0.846 & 0.830 & 0.817 & 0.792 & 0.797 \\
\hline \multirow[t]{3}{*}{ FAST } & RMSD & 0.454 & 0.530 & 0.514 & 0.490 & 0.322 & 0.303 \\
\hline & Align & 0.770 & 0.800 & 0.773 & 0.757 & 0.684 & 0.672 \\
\hline & SN & 0.747 & 0.802 & 0.779 & 0.761 & 0.684 & 0.671 \\
\hline \multirow[t]{2}{*}{ MaxCMO } & Align & 0.665 & 0.685 & 0.687 & 0.730 & 0.672 & 0.667 \\
\hline & Overlap & 0.682 & 0.751 & 0.743 & 0.769 & 0.706 & 0.693 \\
\hline \multirow[t]{3}{*}{ TM-align } & RMSD & 0.475 & 0.624 & 0.602 & 0.550 & 0.354 & 0.336 \\
\hline & Align & 0.695 & 0.747 & 0.733 & $0.74 I$ & 0.656 & 0.645 \\
\hline & $\mathrm{TM}$ & 0.705 & 0.773 & 0.756 & 0.751 & 0.673 & 0.666 \\
\hline USM & USM & 0.678 & 0.686 & 0.680 & 0.683 & 0.578 & 0.566 \\
\hline \multirow[t]{4}{*}{ Consensus } & All & 0.764 & 0.816 & 0.797 & 0.793 & 0.724 & 0.712 \\
\hline & BestOfEach $^{a}$ & 0.759 & 0.818 & 0.804 & 0.803 & 0.746 & 0.740 \\
\hline & Best3b & 0.780 & 0.865 & 0.854 & 0.847 & 0.710 & 0.806 \\
\hline & Best2c & 0.725 & 0.863 & 0.855 & 0.845 & 0.799 & 0.791 \\
\hline
\end{tabular}

optimal combination of different similarity methods is crucial.

\section{Benchmark Tests}

In the previous sections, we have shown the influence of different similarity methods and measures on the quality of the results. Here, we concentrate on their speed (including pre- and post-processing times) in order to show ProCKSI's performance. We have conducted benchmark tests and give the calculation times for the structure comparisons using five different datasets with different numbers of protein chains with six different similarity methods (Table 6 and Table 7). Additionally, the time for the preparation of the contact maps needed for the USM and MaxCMO calculations are given. These include times to parse the PDB files, extract the $\mathrm{C}_{\alpha}$ or $\mathrm{C}_{\beta}$ atoms representing the protein structure, and calculate the distance matrix and contact map for each structure.

The benchmark tests were performed on our mini cluster with 3 dual-processor Intel Xeon/3.2 GHz computers with 4 GB memory. The independent similarity comparison modules were distributed in parallel over all available cluster nodes, making sure that the USM and MaxCMO calculations started not before the contact map preparations had finished. Figure 7 shows ProCKSI's response times for the completion of a request as a function of the size of the corresponding dataset, being represented as the dataset's total number of residues (Figure 7 - middle and bottom). Instead of using the number of chains per dataset only (Figure 7 - top), this measure reflects the size of the entire dataset better, also including the different protein sizes (Table 7).

The USM calculations are almost always the fastest to produce similarity values for an all-against-all structure comparison, but do not give an alignment. For smaller datasets, USM is closely followed by TM-align, whereas FAST beats TM-align for the biggest dataset (RS212). For the latter, performing over 22500 comparisons, USM and FAST return the complete results in about 50 minutes, and TM-align takes less then 85 minutes. This is still more than seven times faster than DaliLite, and ten times faster than CE. The slowest method in our benchmark test is the Max- 
Table 6: Benchmark Tests of ProCKSI: Datasets. Overview of the datasets used for the benchmark tests of ProCKSI, comprising ${ }^{a}$ the first chain of the first model, and $b$ all chains of the first model, respectively. The average number of residues per chain is rounded to the next integer value. The hash symbol (\#) abbreviates Number of.

\begin{tabular}{llcccc}
\hline Dataset & & \# Chains per Dataset & \# Comparisons per Dataset & \# Residues per Dataset & \# Residues per Chain \\
\hline CK34 & {$[17]^{a}$} & 34 & 595 & 6102 & 179 \\
CK53 & {$[17]^{b}$} & 53 & 1431 & 9939 & 188 \\
PK45 & {$[83]^{a}$} & 45 & 1035 & 13360 & 297 \\
PK49 & {$[83]^{b}$} & 49 & 1225 & 12977 & 270 \\
LKR6 & {$[18]^{a}$} & 6 & 21 & 2296 & 383 \\
LKRI5 & {$[18]^{b}$} & 15 & 120 & 4740 & 339 \\
RSI19 & {$[87]^{a}$} & 119 & 7140 & 23053 & 197 \\
RS212 & {$[87]^{b}$} & 212 & 22578 & 39399 & 198 \\
S33 & {$[21]^{a}$} & 33 & 561 & 532 & 168 \\
S73 & {$[21]^{b}$} & 73 & 2701 & 12999 & 178
\end{tabular}

CMO method, which took over two days to complete all comparisons. MaxCMO is implemented as a randomised heuristic algorithm hence requiring for each pair of structures to be compared several restarts as to gain statistical confidence. We used 10 restarts in this benchmark test resulting in up to five times higher calculation times than those of DaliLite. As mentioned before, a higher restart factor provides better chances of getting a better alignment but consumes more computation time.

These benchmark tests show clearly that the time scales the different similarity comparison methods operate on can be quite different. Thus, when producing a consensus similarity result it is important to take both time and quality into account as one might get a reasonable good result with the right combination of fast and reliable comparison methods.

\section{Conclusion and Discussion \\ Summary}

In this paper, we have introduced a new decision-support meta-server for Protein (Structure) Comparison, Knowledge, Similarity and Information (ProCKSI). We have conducted three different experiments with different datasets in order to verify ProCKSI's new Consensus method based on a total-evidence approach. In a first test, we evaluated results from the CASP6 competition using the ProCKSI/ Consensus method, which included all similarity comparison measures using contact maps as their input (USM, MaxCMO/Overlap, MaxCMO/Align). ProCKSI's new Consensus method agrees very well with CASP's GDT-TS method, the community's gold standard. In the few cases where the two methods produced contradictory ranking results, the ProCKSI/Consensus method could detect a better model with a higher similarity (value) and even higher sequence similarity.

In our second experiment, we tested the influence of different combinations of similarity measures on the cluster-

Table 7: Benchmark Tests of ProCKSI: Calculation Times. Calculation times of all datasets with different similarity comparison methods used for the benchmark tests of ProCKSI. For USM and MaxCMO, the calculation times include the preparation times for the contact maps (CM) needed. The datasets are defined in Table 6

\begin{tabular}{cccccccc}
\hline Dataset & CM & USM & FAST & $\begin{array}{c}\text { Times [min] for } \\
\text { TM-align }\end{array}$ & DaliLite & CE & MaxCMO \\
& & & 1.72 & 3.20 & 2.08 & 19.95 & 16.78 \\
CK34 & 0.48 & 4.23 & 5.07 & 4.97 & 50.93 & 48.42 & 21.70 \\
CK53 & 0.73 & 5.83 & 11.93 & 7.90 & 107.67 & 69.03 & 471.70 \\
PK45 & 1.08 & 7.20 & 9.62 & 7.68 & 112.65 & 66.62 & 470.52 \\
PK49 & 1.10 & 1.20 & 1.23 & 1.00 & 3.90 & 1.40 & 19.43 \\
LKR6 & 0.18 & 1.93 & 1.58 & 1.40 & 14.60 & 6.70 & 70.27 \\
LKRI5 & 0.42 & 17.18 & 17.15 & 28.75 & 210.63 & 305.95 & 111.62 \\
RSI19 & 1.77 & 47.18 & 50.07 & 83.05 & 613.70 & 867.52 & 3275.48 \\
RS212 & 3.15 & 1.93 & 1.73 & 1.73 & 22.02 & 8.73 & 76.90 \\
S33 & 0.37 & 4.88 & 6.57 & 8.17 & 124.43 & 51.87 & 419.03 \\
S73 & 0.83 & & & & &
\end{tabular}



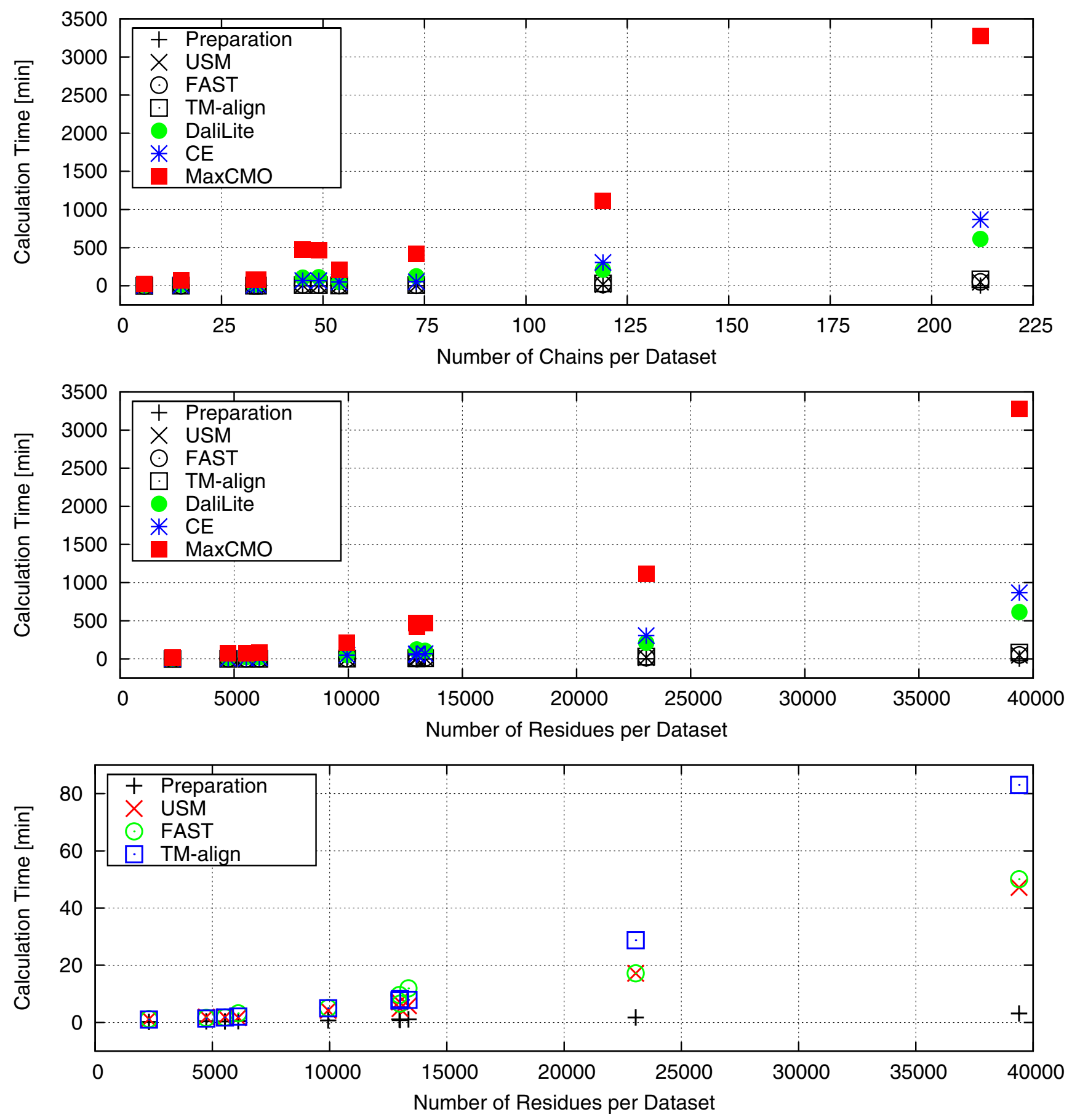

\section{Figure 7}

Benchmark Tests of ProCKSI. ProCKSI's response times for the completion of a request using all available similarity measures as a function of the number of all chains (top) and the number of residues (middle and bottom) in the corresponding dataset. The bottom panel is a magnification of the middle one, displaying only the fastest three methods and the contact map preparation for more clarity. 
ing result of a set of protein kinases. The results using structural similarity comparison methods were compared against the classification scheme by Hanks and Hunter that had been derived from sequence similarities. Again, confirming the findings of our first experiment, none of the similarity methods completely reproduced the original classification when taken separately. ProCKSI's Consensus method, on the other hand, using just USM and DaliLite/Z, was able to reproduce the correct clustering according to Hanks and Hunter.

In our third experiment, we analysed the quality and performance of six different protein comparison methods as provided by ProCKSI by means of Receiver Operator Characteristics (ROC). Using the Rost and Sander dataset, the Area Under the Curve (AUC) values were employed in order to compare the results against SCOP's hierarchical classification levels as the gold standard. We investigated different combinations and sets of similarity measures in order to produce the best consensus measure. Surprisingly, combining the best three measures for SCOP's Class, Fold, Family and Species levels, or the best two measures for the remaining levels, respectively, we obtain higher AUC values than any of the contributing similarity measures by themselves. This synergistic effect shows that ProCKSI's new Consensus measure can outperform for some datasets even well established and reliable similarity comparison measures such as DaliLite and CE.

Additionally, we also benchmarked ProCKSI on various other datasets in terms of compute time, and found it competitive with current state of the art.

\section{Discussion}

ProCKSI implements several different similarity methods and allows the user to provide results from his/her own similarity assessment, which are treated equally to ProCKSI's own results. When trying to produce a consensus similarity result it is important to take both time and quality into account as one might get a reasonable good result with the right combination of fast and reliable comparison methods. For example, other web servers for protein structure comparison (e.g. DALI, CATH, LGA, CE, FATCAT, FAST, etc.) allow only pairs of proteins to be compared or they compare one given protein structure against a database of pre-calculated/pre-aligned structures. In the latter case, the result of such a comparison might be delivered almost instantaneously, whereas the response time for a pairwise comparison or arbitrary proteins depends on the following factors: a) the algorithms used to make the comparison, b) the sizes of the proteins to be compared, and c) the servers' load and internet traffic. When comparing a set of $N$ proteins against each other, there are $\frac{N^{2}+N}{2}$ different combinations of pairs to be calculated, assuming that the comparison of protein $p_{1}$ with protein $p_{2}$ gives the same result as comparing $p_{2}$ with $p_{1}$.

In addition to the algorithms' complexity and the number of protein pairs to be compared when calculating the similarity of a set of proteins with a specific comparison server that allows only pairwise comparisons, each pair has to be generated and uploaded separately, and the desired models and chains have to be selected/extracted repeating this procedure for the same protein file more than once. After submitting the job, it has to be checked periodically until results are available, which then must be downloaded separately. Finally, the results would have to be integrated manually in order to produce a similarity matrix for all proteins in the set. This can be tedious and error prone, especially when dealing with sets of tens or hundreds of structures. ProCKSI, on the other hand, helps to minimise the data management overhead by preparing the entire dataset once in a few steps, by giving access to a variety of similarity methods and measures in one easy-to-use interface, by keeping track of the progress of all calculations, and by seamlessly and automatically integrating all results. That is, ProCKSI hides from the end user the complexity behind a systematic comparison studies.

As our experiments have shown, not all comparison methods perform equally well on all datasets. MaxCMO, for instance, gave excellent results in our CASP experiment, but could discriminate the Kinases only partially. The important lesson here is not that MaxCMO performed poorly on the Kinases dataset (as we mentioned in the introduction that every method has an Achilles heel), but rather that even when adding to the consensus a method that discriminates the dataset fairly poorly, one can still obtain comparably good results. These findings lend support to our integrative approach of combining various similarity measures thus producing a robust consensus similarity, and show that the best results potentially do prevail even when adding "noise" to the data. This is a particular relevant observation as in general the biologist, faced with a given dataset, does not know a priori which method to use. Hence, he/she would be on safer grounds if he/she was to use all of the available methods (through a decision support system such as ProCKSI) and rely on a consensus method.

We have also found that there are different optimal combinations of different methods when generating the consensus similarity picture for different datasets. Hence, finding a good set and combination of similarity compar- 
ison methods for a given dataset remains a key open question.

\section{Future Work}

In the future, we plan to extend ProCKSI integrating other similarity methods and link to further databases, e.g. $[94,95]$, and systematically investigate the impact of different compressors in the USM [96].

In order to cope with the vast amount of calculations and data, we will seek to enhance our computational platform by recruiting more compute servers, by utilising established web services for protein comparison, and by deploying the calculations to the GRID.

More importantly, we will investigate new and more intelligent ways of computing consensus similarities using e.g. machine learning techniques [97], and integrate automated cluster validation techniques, e.g. [98,99]. A measure of variance such as averaged ROC curves from bootstrapping or cross-validation with a variety of different datasets is needed in order to give a final conclusion about the optimal set of comparison methods [86]. This at hand, we will be able to give the user more and better advice and guidelines of which methods to use for a particular problem.

Additionally, we plan to integrate into ProCKSI a second analysis strategy using average consensus trees and supertrees $[100,101]$ so as to complement our current total-evidence approach $[47,102,103]$.

\section{Availability and Requirements Project name: ProCKSI}

Project home page: http://www.procksi.net

Operating system(s): Linux (back-end), platform independent (front-end)

Programming languages: PERL, Java, C++

Other requirements: Web Browser, Java Runtime Environment (JRE), JavaScript, Cascading Style Sheets (CSS)

License: Web server freely available without registration

Restrictions to use by non-academics: on request

\section{List of Abbreviations}

AUC : Area Under the Curve;

CASP : Critical Assessment of Techniques for Protein Structure Prediction;
CE : Combinatorial Extension of the optimal path;

CL : Complete Linkage;

CM : Contact Map;

DALI : Distance Matrix Alignment;

DM : Distance Matrix;

FAST : FAST Alignment and Search Tool;

FPr : False Positive rate;

GDT-TS : Global Distance Test - Total Score;

HH : Hanks and Hunter;

LGA : Local Global Alignment;

MaxCMO : Maximum Contact Map Overlap;

NCD : Normalised Compression Distance;

PDB : Protein Data Bank;

PK : Protein Kinase;

PKR : Protein Kinase Resource;

ProCKSI : Protein (Structure) Comparison, Knowledge, Similarity and Information;

RMSD : Root Mean Square Distance;

ROC : Receiver Operator Characteristics;

SCOP : Structural Classification Of Proteins;

SDA : Sequence Dependent Analysis;

SIA : Sequence Independent Analysis;

SL : Single Linkage;

SM : Similarity Matrix;

SSM : Standardised Similarity Matrix;

TM : Template Modelling;

TPr : True Positive rate;

UPGMA : Unweighted Pair Group Method with Arithmetic mean; 
USM : Universal Similarity Metric;

VRML : Virtual Reality Markup Language;

WMV : Ward's Minimum Variance.

\section{Authors' contributions}

DB: software design, implementation, writing, assessment. NK: project conception, software design, writing, assessment, funding. JDH: writing, discussions, funding. JB: writing, discussions. EKB: writing, funding. All authors read and approved the final manuscript.

\section{Acknowledgements}

Natalio Krasnogor acknowledges the Biotechnology and Biological Sciences Research Council (BBSRC) for grant BB/C5II764/I and the Engineering and Physical Sciences Research Council (EPSRC) for grants GR/T07534/0I and EP/ D06/57I/I. Jacek Blazewicz acknowledges a grant from the Ministry of Higher Education in Poland. We thank Benjamin Bulheller, David Pelta and Juan-Ramón Gonzáles Gonzáles for their ongoing collaboration within the ProCKSI project, and Pooja Jain for useful feedback on the web server. We thank the reviewers for their valuable comments.

\section{References}

I. Koehl P: Protein structure similarities. Curr Opin Struct Biol 200I, I I:348-353.

2. Kryshtafovych A, Milostan M, Szajkowski L, Daniluk P, Fidelis K: CASP6 Data Processing and Automatic Evaluation at the Protein Structure Prediction Center. Proteins Struct Funct Bioinf 2005:19-23.

3. Ferro D, Hermans J: A Different Best Rigid-body Molecular Fit Routine. Acta Crystallogr 1977, A33:345-347.

4. Kabsch W: A Discussion of the Solution for the Best Rotation to Relate Two Sets of Vectors. Acta Crystallogr 1978, A34:827-828.

5. Vriend G, Sander C: Detection of common three-dimensional substructures in proteins. Proteins 1991, I I:5।-58.

6. Alexandrow N, Takahashi K, Go N: Common spatial arrangements of backbone fragments in homologous and nonhomologous proteins. J Mol Biol 1992, 225:5-9.

7. Fischer D, Bachar O, Nussinov R, Wolfson H: An efficient automated computer vision based technique for detection of three-dimensional structural motifs in proteins. J Biomol Struct Dyn 1992, 9(4):769-789.

8. Holm L, Sander C: Protein Structure Comparison by Alignment of Distance Matrices. J Mol Biol 1993, 233:123-138.

9. Artymiuk PJ, Poirrett AR, Rice DW, Willet P: The use of graph theoretical methods for the comparison of the structure of biological macromolecules. Top Curr Chem 1995, 174:73-103.

10. Wu T, SC S, Hastie T, DL B: Regression analysis of multiple protein structures. J Comput Biol 1998, 5:585-595.

II. Shindyalov I, Bourne P: Protein structure alignment by incremental combinatorial extension (CE) of the optimal path. Protein Eng 1998, I I:739-747.

12. Zemla A: LGA program: a method for dinding 3D similarities in protein structures. Nucleic Acids Res 2003, 31:3370-3374.

13. Taylor WR: Protein structure comparison using iterated double dynamic programming. Protein Sci 1999, 8:654-665.

14. Gerstein M, Levitt M: Comprehensive assessment of automatic structural alignment against a manual standard: the SCOP classification of proteins. Protein Sci 1998, 7:445-456.

15. Yang A, Honig B: An integrated approach to the analysis and modeling of protein sequences an structures. I. Protein structural alignment and a quantitative mesasure for protein structural distance. J Mol Biol 2000, 301:665-678.

16. Szustakowski J, Weng Z: Protein structure alignment using genetic algorithm. Proteins 2000, 38:428-440.
17. Chew LP, Kedem K: Finding the consensus shape for a protein family. In Proceedings of the I 8th Annual Symposium on Computational Geometry (SCG) New York: Springer; 2002:64-73.

18. Leluk J, Konieczny L, Roterman I: Search for structural similarity in proteins. Bioinformatics 2003, 19:117-124.

19. Goldman D, Papadimitriou C, Istrail S: Algorithmic Aspects of Protein Structure Similarity. In Proceedings of the 40th Annual Symposium on Foundations of Computer Science Washington: IEEE Computer Society; 1999:5I2-52I.

20. Lancia G, Carr R, Walenz B, Istrail S: IOI optimal pdb structure alignments: a branch-and-cut algorithm for the maximum contact map overlap problem. In Proceedings of the 5th Annual Interantional Conference on Computational Molecular Biology (RECOMB) New York: ACM Press; 200I:192-202.

21. Caprara A, Lancia G: Structural alignment of large-size proteins via lagrangian relaxation. In Proceedings of the 6th Annual Conference on Research in Computational Molecular Biology (RECOMB) New York: ACM Press; 2002:100-108.

22. Carr B, Hart W, Krasnogor N, Burke EK, Hirst JD, Smith J: Alignment of protein structures with a memetic evolutionary algorithm. In Proceedings of the Genetic and Evolutionary Computation Conference (GECCO) San Francisco: Morgan Kaufmann; 2002:1027-1034.

23. Caprara A, Carr R, Istrail S, Lancia G, Walenz B: I00I Optimal PDB Strurcture Alignments: Integer Programming Methods for Finding the Maximum Contact Map Overlap. J Comput Biol 2004, I I:27-52

24. Krasnogor N, Pelta DA: Measuring the similarity of protein structures by means of the universal similarity metric. Bioinformatics 2004, 20:1015-1021.

25. Pelta DA, Krasnogor N, Bousono-Calzon C, Verdagay JL, Hirst JD Burke E: A fuzzy sets based generalization of contact maps for the overlap of protein structures. Fuzzy Sets and Systems 2005, I52:102-123.

26. Krissinel E, Henrick K: Secondary-structure matching (SSM), a new tool for fast protein structure alignment in three dimensions. Acta Crystallogr D Biol Crystallogr 2004, 60(Pt I2 Pt I):2256-2268

27. Krasnogor N: Self-Generating Metaheuristics in Bioinformatics: The Protein Structure Comparison Case. Genetic Programming and Evolvable Machines 2004, 5: I8I-201

28. Zhu J, Z W: FAST: A novel protein structure alignment algorithm. Proteins Struct Funct Bioinf 2005, 58:618-627.

29. Strickland D, Barnes E, Sokil J: Optimal Protein Structure Alignment Using Maximum Cliques. Opterations Research 2005, 53:389-402.

30. Shatsky M, Nussinov R, Wolfson H: Flexible protein alignment and hinge detection. Proteins Struct Funct Genet 2002, 48:242-256.

31. Ye Y, Godzik A: FATCAT: a web server for flexible structure comparison and structure similarity searching. Nucleic Acids Res 2004, 32:W582-W585.

32. Vesterstrom J, Taylor W: Flexible Secondary Structure Based Protein Structure Comparison Applied to the Detection of Circular Permutation. J Comput Biol 2006, 13:43-63.

33. The Bioinformatics Links Directory [http://bioinformatics.ca/ links directory/?subcategory id=136]

34. Galperin M: The Molecular Biology Database Collection: 2004 Update. Nucleic Acids Res 2004, 32:D3-D22.

35. Galperin MY: The Molecular Biology Database Collection: 2005 Update. Nucleic Acids Res 2005, 33:D5-D24.

36. Galperin M: The Molecular Biology Database Collection: 2006 update. Nucleic Acids Res 2006, 34:D3-D5.

37. Webserver Issue. Nucleic Acids Res 2005, 33:WI-W786.

38. Webserver Issue. Nucleic Acids Res 2006, 34:WI-W75I.

39. Database Issue. Nucleic Acids Res 2004, 32:DI-D599.

40. Database Issue. Nucleic Acids Res 2005, 33:DI-D679.

41. Database Issue. Nucleic Acids Res 2006, 34:DI-D784

42. Database Issue. Nucleic Acids Res 2007, 35:DI-D910.

43. Camoglu $O$, Can T, Singh A: Integrating multi-attribute similarity networks for robust representation of the protein space. Bioinformatics 2006, 22: $1585-1592$

44. Filkov V, Skiena S: Heterogeneous Data Integration with the Consensus Clustering Formalism. In Proceedings of the Ist International Workshop on Data Integration in the Life Science (DILS) LNCS Berlin: Springer; 2004:1 10-123. 
45. Li M, Chen X, Li X, Vitányi PMB, Ma B: The Similarity Metric. IEEE Trans Inf Theor 2004, 50:3250-3264.

46. Fischer D, Rychlewski L, Dunbrack RL Jr, Ortiz AR, Elofson A: Servers for protein structure prediction. Curr Opin Struct Biol 2006, 16:178-182.

47. Lapointe FJ, Kirsch J, Hutcheon J: Total Evidence, Consensus, and Bat Phylogeny: A Distance-Based Approach. Mol Phylogenet Evol 1999, I I:55-66.

48. Kocsor A, Kertesz-Farkas A, Kajan L, Pongor S: Application of compression-based distance measures to protein sequence classification: a methodological study. Bioinformatics 2006, 22:407-4I2.

49. Holm L, Park J: DaliLite workbench for protein structure comparison. Bioinformatics 2000, I 6:566-567.

50. Zhang $Y$, Skolnick J: TM-align: A protein structure alignment algorithm based on TM-score. Nucleic Acids Res 2005, 33:2302-2309.

5I. Bingham J, Sudarsanam S: Visualizing large hierarchical clusters in hyperbolic space. Bioinformatics 2000, I 6:660-66I.

52. Berman H, Westbrook J, Feng Z, Gilliland G, Bhat T, Weissig H, Shindyalov I, Bourne P: The Protein Data Bank. Nucleic Acids Res 2000, 28:235-242.

53. Hubbard TJ, Ailey B, Brenner SE, Murzin AG, Chothia C: SCOP: a Structural Classification of Proteins database. Nucleic Acids Res 1999, 27:254-256.

54. Andreeva A, Howorth D, Brenner SE, Hubbard TJP, Chothia C, Murzin AG: SCOP database in 2004: refinements integrate structure and sequence family data. Nucleic Acids Res 2004, 32:D226-D229.

55. Orengo CA, Michie AD, Jones S, Jones DT, Swindells MB, Thornton JM: CATH - A Hierarchic Classification of Protein Domain Structures. Structure 1997, 5:1093-I I08.

56. Pearl F, Todd A, Sillitoe I, Dibley M, Redfern O, Lewis T, Bennett C, Marsden R, Grant A, Lee D, Akpor A, Maibaum M, Harrison A, Dallman T, Reeves G, Diboun I, Addou S, Lise S, Johnston C, Sillero A, Thornton J, Orengo C: The CATH Domain Structure Database and related resources Gene3D and DHS provide comprehensive domain family information for genome analysis. Nucleic Acids Res 2005, 33:D247-D25I.

57. Hoffmann R, Valencia A: A Gene Network for Navigating the Literature. Nat Genet 2004, 36:664-664.

58. Hoffmann R, Valencia A: Implementing the iHOP concept for navigation of biomedical literature. Bioinformatics 2005, 2 I (Suppl 2):ii252-ii258.

59. Cilibrasi R, Vitanyi MB: Clustering by Compression. IEEE Trans Inf Theor 2005, 5 I: I523-I545.

60. Vendruscolo M, Najmanovich R, Domany E: Protein Folding in Contact Map Space. Phys Rev Lett 1999, 82:656-659.

61. Gelly JC, de Brevern AG, Hazout S: Protein Peeling: an approach for splitting a 3D protein structure into compact fragments. Bioinformatics 2006, 22:129-133.

62. Margara L, Vassura M, Di Lena P, Medri F, Fariselli P, Casadio R: Reconstruction of 3D Structures From Protein Contact Maps. In Proceedings of the 3rd International Symposium on Bioinformatics Research and Applications (ISBRA), LNBI 4463 Berlin: Springer; 2007:578-589.

63. Berrera M, Molinari H, Fogolari F: Amino acid empirical contact energy definitions for fold recognition in the space of contact maps. BMC Bioinformatics 2003, 4:8.

64. Punta M, Rost B: PROFcon: novel prediction of long-range contacts. Bioinformatics 2005, 2 1:2960-2968.

65. Graña O, Eyrich VA, Pazos F, Rost B, Valencia A: EVAcon: a protein contact prediction evaluation service. Nucleic Acids Res 2005, 33:W347-W35I.

66. Graña O, Baker D, MacCallum RM, Meiler J, Punta M, B R, Tress ML, Valencia A: CASP6 assessment of contact prediction. Proteins Struct Funct Bioinf 2005, 61:214-224.

67. Chung JL, Beaver JE, Scheeff ED, Bourne PE: Con-Struct Map: A Comparative Contact Map Analysis Tool. Bioinformatics 2007, 23:249|-2492

68. Kraulis PJ: MOLSCRIPT: A Program to Produce Both Detailed and Schematic Plots of Protein Structures. J Appl Cryst I99I, 24:946-950.

69. Sokal RR, Michener CD: A statistical method for evaluating systematic relationships. Univ Kansas Sci Bull 1958, 38: 1409-|438.
70. Ward J Jr: Hierarchical Grouping to Optimize an Objective Function. J Amer Statist Assoc 1963, 58:236-244.

71. Clustering Calculator [http://www2.biology.ualberta.ca/jbrzusto/ cluster.php]

72. Felsenstein J: PHYLIP - Phylogeny Inference Package (Version 3.2). Cladistics 1989, 5:164-166.

73. Hanks S, Hunter T: The eurkaryotic protein kinase superfamily: kinase (catalytic) domain structure and classification. The FASEB Journal 1995, 9:576-596.

74. Fischer D, Rychlewski L, Dunbrack RL Jr, Ortiz AR, Elofson A: CAFASP3: the third critical assessment of fully automated structure prediction methods. Proteins 2003, 53(Suppl 6):503-516.

75. Tress M, Ezkurdia I, Graña O, López G, Valencia A: Assessmentof Predictions Submitted for the CASP6 Comparative Modeling Category. Proteins Struct Funct Bioinf 2005:27-45.

76. Valencia A, Lee B, Dunbrack RL Jr: Domain definition and target classification for CASP6. Proteins 2005, 6 I (Suppl 7):8-I8.

77. Petretti C, Prigent C: The Protein Kinase Resource: everything you always wanted to know about protein kinases but were afraid to ask. Biol Cell 2005, 97: I I3- I 18.

78. Smith $C$ : The protein kinase resource and other bioinformation resources. Prog Biophys Mol Biol 1999, 71:525-533.

79. Cheek S, Zhang H, Grishin N: Sequence and structure classification of Kinases. J Mol Biol 2002, 320:855-88I.

80. Manning G, Whyte D, Martinez R, Hutner T, Sudarsanam S: The Protein Kinase Complement of the Human Genome. Science 2002, 298: $1912-1934$.

8I. Cheek S, Ginalski K, Zhang H, Grishin N: A comprehensive update of the sequence an structure classification of kinases. BMC Struct Biol 2005, 5:6.

82. Fernandez-Fuentes N, Hermoso A, Espandaler J, Querol E, Aviles F, Oliva B: Classification of Common Functional Loops of Kinase Super-Families. Proteins 2004, 56:539-555.

83. Mirror of the Protein Kinase Resourse (PKR) [http:// www.nih.go.jp/mirror/Kinases]

84. Smith C, Shindyalov I, S V, Gribskov M, Taylor S, Ten Eyok L, P B: The Protein Kinase Resource. Trends Biochem Sci 1997, I I:444-446.

85. SCOP: Structural Classification of Proteins [http://scop.mrc-
[ Imb.cam.ac.uk/scop]. [Release I.69]

86. Fawcett T: Introduction to ROC analysis. Pattern Recog Lett 2006, 27:86I-874.

87. Rost B, Sander C: Prediction of protein secondary structure at better than 70\% accuracy. J Mol Biol 1993, 232:584-599.

88. Hou J, Jun SR, Zhang C, Kim SH: Global mapping of the protein structure space and application in structure-based inference of protein function. Proc Natl Acad Sci USA 2005, I 02:365 I-3656.

89. Egan J: Signal detection theory and ROC analysis. In Series in Cognition and Perception New York: Academic Press; 1995.

90. Spackman K: Signal detection theory: Valuable tools for evaluating inductive learning. In Proceedings of the 6th International Workshop on Machine Learning Volume 283. San Francisco: Morgan Kaufman; 1989:160-163.

91. Receiver Operating Characteristic (ROC) Literature Research 2007 [http://splweb.bwh.harvard.edu:8000/pages/ppl/zou/ roc.html]. Active link not available; last accessed 26th Oct

92. Leplae R, Hubbard T: MaxBench: evaluation of sequence and structure comparison methods. Bioinformatics 2002, I 8:494-495.

93. Kolodny R, Koehl P, Levitt M: Comprehensive Evaluation of Protein Structure Alignment Methods: Scoreing by Beometric Measures. J Mol Biol 2005, 346: I I73- I I88.

94. Portugaly E, Harel A, Linial N, Linial M: EVEREST: Automatic identification and classification of protein domains in all protein sequences. BMC Bioinformatics 2006, 7:277.

95. Portugaly E, Linial N, Linial M: EVEREST: A collection of evolutionary conserved protein domains. Nucleic Acids Res 2007, 35:D24I-D246.

96. Ferragina P, Giancarlo R, Greco V, Manzini G, Valiente G: Compression-based classification of biological sequences and structures via the Universal Similarity Metric: experimental assessment. BMC Bioinformatics 2007, 8:252.

97. Stout M, Bacardit J, Hirst J, Smith R, Krasnogor N: Prediction of Topological Contacts in Proteins Using Learning Classifier Systems. Soft Comput J in press. 
98. Varshavsky R, Linial M, Horn D: COMPACT - A Comparative Package for Clustering Assessment. In Proceedings of the ISPA Workshops, LNCS:3759 Berlin: Springer; 2005:159-167.

99. Handl J, Knowles J, Kell D: Computational cluster validation in post-genomic data analysis. Bioinformatics 2005, 21:320I-32I 2 .

100. Munzner T, Guimbretière F, Tasiran S, Zhang L, Zhou Y: TreeJuxtaposer: Scalable Tree Comparison using Focus+Context with Guaranteed Visibility. ACM Transaction on Graphics 2003, 22:453-462.

10I. Lapointe FJ, Cucumel G: The Average Consensus Procedure: Combination of Weighted Trees Containing Identical or Overlapping Sets of Taxa. Syst Biol 1997, 46:306-3/2.

102. Lapointe JF, Wilkinson M, Bryant D: Matrix Representations with Parsimony or with Distances: Two Sides of the Same Coin? Syst Biol 2003, 52:865-868.

103. Levasseur C, Lapointe F): Total Evidence, Average Consensus and Matrix Representation with Parsimony: What a Difference Distances Make. Evol Bioinf Online 2006, 2:249-253.

Publish with Biomed Central and every scientist can read your work free of charge

"BioMed Central will be the most significant development for disseminating the results of biomedical research in our lifetime. "

Sir Paul Nurse, Cancer Research UK

Your research papers will be:

- available free of charge to the entire biomedical community

- peer reviewed and published immediately upon acceptance

- cited in PubMed and archived on PubMed Central

- yours - you keep the copyright

Submit your manuscript here:

http://www.biomedcentral.com/info/publishing_adv.asp
BioMedcentral 